\title{
Exact solutions of domain wall junctions in arbitrary dimensions
}

\author{
Minoru Eto $\odot,{ }^{1,2, *}$ Masaki Kawaguchi $\odot,{ }^{1, \dagger}$ Muneto Nitta, ${ }^{2,3, \$}$ and Ryotaro Sasaki ${ }^{1}$ \\ ${ }^{1}$ Department of Physics, Yamagata University, Kojirakawa-machi 1-4-12, Yamagata, \\ Yamagata 990-8560, Japan \\ ${ }^{2}$ Research and Education Center for Natural Sciences, Keio University, Hiyoshi 4-1-1, \\ Yokohama, Kanagawa 223-8521, Japan \\ ${ }^{3}$ Department of Physics, Keio University, Hiyoshi 4-1-1, Yokohama, Kanagawa 223-8521, Japan
}

(Received 21 April 2020; accepted 17 August 2020; published 8 September 2020)

\begin{abstract}
Exact analytic solutions of static, stable, nonplanar Bogomol'nyi-Prasad-Sommerfield (BPS) domain wall junctions are obtained in extended Abelian-Higgs models in $(D+1)$-dimensional spacetime. For specific choice of mass parameters, the Lagrangian is invariant under the symmetric group $\mathcal{S}_{D+1}$ of degree $D+1$ spontaneously broken down to $\mathcal{S}_{D}$ in vacua, admitting $\mathcal{S}_{D+1} / \mathcal{S}_{D}$ domain wall junctions. In $D=2$, there are three vacua and three domain walls meeting at a junction point, in which the conventional topological charges $Y$ and $Z$ exist for the BPS domain wall junctions and the BPS domain walls, respectively, as known before. In $D=3$, there are four vacua, six domain walls, four junction lines on which three domain walls meet, and one junction point on which all the six domain walls meet. We define a new topological charge $X$ for the junction point in addition to the conventional topological charges $Y$ and $Z$. In general dimensions, we find that the configuration expressed in the $D$-dimensional real space is dual to a regular $D$-simplex in the $D$-dimensional internal space and that a $d$-dimensional subsimplex of the regular $D$-simplex corresponds to a $(D-d)$-dimensional intersection. Topological charges are generalized to the level- $d$ wall charge $W_{d}$ for the $d$-dimensional subsimplexes.
\end{abstract}

DOI: 10.1103/PhysRevD.102.065006

\section{INTRODUCTION}

Domain walls (or kinks) are the simplest topological solitons separating discrete vacua or ground states [1-3], often created in phase transitions associated with spontaneous breakings of discrete symmetries [4,5] in various systems from small to large such as magnets [6], graphenes [7], carbon nanotubes, chiral p-wave superconductors [8], Bose-Einstein condensations of ultracold atomic gases [9], helium superfluids [10-12], nuclear matter [13,14], as well as quark matter [15] relevant for interior of neutron stars, and our Universe $[4,16]$. In cosmology, if they appear at a phase transition in the early Universe, then there happens the so-called domain wall problem [16], that is, the domain wall energy dominates Universe to make it collapse. However, if the tension of the domain walls is sufficiently low, cosmological domain wall networks are allowed and are suggested as a candidate of dark matter and/or dark

\footnotetext{
meto@sci.kj.yamagata-u.ac.jp †ddwbb.daigaku@gmail.com

\#nitta@phys-h.keio.ac.jp
}

Published by the American Physical Society under the terms of the Creative Commons Attribution 4.0 International license. Further distribution of this work must maintain attribution to the author(s) and the published article's title, journal citation, and DOI. Funded by SCOAP . energy [17]. In helium superfluids, such domain walls are created in a similar manner, thereby simulating cosmological phase transitions $[11,12]$.

On the other hand, it is widely known that supersymmetry (SUSY) is very intimate notion with various topological solitons such as domain walls, vortices, monopoles, and instantons etc. [18]. As one of fascinating features, SUSY allows for topological solitons to be the so-called Bogomol'nyi-Prasad-Sommerfield (BPS) states [19,20], which attain the minimum energy for a fixed boundary condition or topology. The BPS solitons satisfy first order differential equations, the so-called BPS equations, rather than equations of motion which are of second order differential equations, and they preserve a fraction of SUSY. Their topological charges are directly connected to central charges of SUSY algebras. The BPS domain walls in 3+1-dimensional spacetime have been extensively studied in $\mathcal{N}=1$ SUSY theories [21-36] and $\mathcal{N}=$ 2 SUSY theories [37-54] (see Refs. [18,55-57] as a review). They preserve a half of SUSY and thereby are called $\frac{1}{2}$ BPS states, and the corresponding SUSY central (tensorial) charge $[23,25,58]$ is conventionally expressed as $Z$. In the models with three or more discrete vacua, there can appear multiple domain walls. The multiple domain walls remain as the $\frac{1}{2}$ BPS states as long as they are all parallel. 
In general, it is more natural that the multiple domain walls are not parallel. If all the domain walls have one spatial dimension (say the $z$-axis) in common, domain walls extend to two-dimensional (2D) space (the $x-y$ plane). The domain walls meet at a line to form a domain wall junction. We call these two-dimensional (of codimensions two) configurations planar domain wall junctions. In SUSY models, the planar domain wall junctions preserve a quarter of supersymmetry [59-61]. They are called the $\frac{1}{4}$ BPS states and are accompanied with a junction topological charge $Y$ in addition to $Z_{m}(m=1,2) .{ }^{1}$ The $\frac{1}{4}$ BPS domain wall junctions have been also studied in $\mathcal{N}=1$ SUSY models $[33,71-79]$ and $\mathcal{N}=2$ SUSY models [80-89]. The domain wall junctions are similar to vortex strings, but the junction charge $Y$ was found to negatively contribute to the total energy [73-75] in contrast to the domain wall charge $Z_{1,2}$ always contributing positively to the total energy. Thus, the junction charge $Y$ should be understood as a sort of binding energy bonding the domain walls [73,75] rather than independent topological solitons. Planer network of the domain walls and junctions were studied as non-BPS states in Refs. [90,91]. Then, it was found that the $\mathcal{N}=2$ SUSY gauge models can have any kind of planar domain wall networks as the $\frac{1}{4}$ BPS states [81,82], similarly to D-brane networks $[92,93]$.

In this paper, we study nonplanar BPS domain wall junctions in which three or more domain walls having angles meet at a point. Namely, we consider the domain wall junctions which are essentially $D$-dimensional in $D+1$-dimensional spacetime for $D \geq 3$. The planar domain wall junctions are $N$-pronged junctions $(N \geq 3)$ of codimension two typically appearing when the models under consideration possess a $\mathbb{Z}_{N}$ symmetry spontaneously broken in the vacua; see, for example, [59,60,71,72,74]. Note that the $\mathbb{Z}_{N}$ symmetry is an Abelian group naturally associated with a discrete rotation group of a regular $N$-gon in two dimensions. The $\mathbb{Z}_{N}$ is a subgroup of $S O(2)$ rotation of the two-dimensional space.

In this work, we generalize this to the higher dimensions. A symmetry group preserving a $D$-dimensional object $(D \geq 3)$ is usually non-Abelian since it is a subgroup of $S O(D)$. For instance, the symmetry group of a regular tetrahedron is the symmetric group of degree four $\mathcal{S}_{4}$ which is non-Abelian. Inspired by the $\mathcal{N}=2$ SUSY QED in $3+$ 1 dimensions, we study a $U(1)$ gauge theory coupled with $N_{F}$ charged scalars and $N_{F}^{\prime}$ reals scalars in $(D+1)$ dimensional spacetime. It turns out that the vacuum structure of the model is indeed $N_{F}^{\prime}$ dimensional. Then, to obtain BPS $D$-dimensional domain wall junctions, we set

\footnotetext{
${ }^{1}$ In $\mathcal{N}=2$ SUSY theories, there are other $\frac{1}{4}$ BPS states, vortex strings ending on domain walls called D-brane solitons [56,62-67], instantons inside vortices [68], and intersecting vortices [69]. See Ref. [70] for classification of all possible $\frac{1}{4}$ and $\frac{1}{8}$ BPS solitons.
}

$N_{F}^{\prime}=D$ and derive the BPS equations. Interestingly, the BPS equations involve arbitrary $D$ signs $\xi_{m}= \pm 1$ $(m=1,2, \ldots, D)$. Therefore, there exist $2^{D}$ different sets of the BPS equations according to choices of $\xi_{m}$. When $D=2$, there are four sets and we find they are identical to four sets of $\frac{1}{4}$ BPS equations in $\mathcal{N}=2$ SQED studied in Refs. [80-86]. In the SUSY context, the number four comes from the number of way of selecting two among eight supercharges.

The main result of this paper is to present exact analytic solutions of nonplanar domain wall junctions in generic $D+1$-dimensional spacetime. For this purpose, we will restrict ourself to the models of $N_{F}=D+1$ with the largest symmetry, that is the symmetric group $\mathcal{S}_{D+1}$. The previously known exact solution of the three-pronged planar domain wall junction [80] corresponds to the case of $D=2$. In the vacua, $\mathcal{S}_{3}$ is spontaneously broken to $\mathcal{S}_{2}$, so that the vacuum structure is the coset $\mathcal{S}_{3} / \mathcal{S}_{2}$ consists of three elements. We then construct a novel exact solution of the three-dimensional domain wall junction connecting the four different vacua for $D=3$. The model has the $\mathcal{S}_{4}$ symmetry spontaneously broken to $\mathcal{S}_{3}$. The vacua are the coset $\mathcal{S}_{4} / \mathcal{S}_{3}$ with four elements. Reflecting the fact that the symmetric group $\mathcal{S}_{4}$ is the symmetry group of the regular tetrahedron, the vacua correspond to four vertices of the tetrahedron. The configuration in the real space has the same symmetric property $\mathcal{S}_{4} / \mathcal{S}_{3}$ as the vacua structure; it consists of six domain walls. Two domain walls arbitrary chosen from them glue along a line junction. There exist four such junction lines, and all the junction lines meet at one point. Correspondingly, the topological charges of the domain walls and domain wall junction lines are $Z_{m}$ and $Y_{m n}(m, n=1,2,3$ and $m>n)$, respectively, as before. In addition, there is a new topological charge $X$ for the junction point. Although the topological charges $Z_{m}$ and $Y_{m n}$ contribute to the energy density, the new topological charge $X$ does not. We then construct an exact solution of a BPS $\mathcal{S}_{D+1} / \mathcal{S}_{D}$ domain wall junction in $D+1$-dimensional spacetime. We find several geometric properties of the solution. The $\mathcal{S}_{D+1} / \mathcal{S}_{D}$ domain wall junction expressed in the real space is dual to a regular $D$-simplex in the internal space whose $D+1$ vertices correspond to the vacua. A $d$-face, a $d$-dimensional subsimplex $(0 \leq d \leq D)$ of the $D$-simplex (the 0 -faces are the vertices, the 1 -faces are the edges, and so on) are dual to $(D-d)$-dimensional building blocks of the configuration. For example, the 0 -faces are dual to the $D$-dimensional vacuum domains, the 1 -faces to the $(D-1)$-dimensional domain walls, and so on. For each $d$, we define a topological charge $W_{d}$ of the level- $d$ $\left(W_{1, m}=Z_{m}, W_{2, m n}=Y_{m n}\right.$, and $W_{3, l m n}=X_{l m n}$, and so on $)$. The symmetric group $\mathcal{S}_{D+1}$ which is the symmetry group of the regular $D$-simplex is isomorphic to the Coxeter group of the type $A_{D}$. The orthographic projection in twodimensional plane of the $D$-simplex is known as the Coxeter plane of type $A_{D}$. We find that the exact solution 
of the $\mathcal{S}_{D+1} / \mathcal{S}_{D}$ domain wall junction provides the Coxeter plane of the type $A_{D}$ when it is expressed in a twodimensional internal space.

This paper is organized as follows. In Sec. II, we present our model, its symmetry structure and vacua. In Sec. III, we derive BPS equations for domain wall junctions. In Sec. IV, we construct a planar $\mathcal{S}_{3} / \mathcal{S}_{2}$ domain wall junction in $D=2$, which is essentially a review of Ref. [80]. In Sec. V, we construct a $\mathcal{S}_{4} / \mathcal{S}_{3}$ domain wall junction in $D=3$. In Sec. VI, they are generalized to $\mathcal{S}_{4} / \mathcal{S}_{3}$ domain wall junctions in $D$ dimensions, and geometric properties are discussed. Section VII is devoted to a summary and discussion. The Appendix summarizes explicit expression of the symmetric group $\mathcal{S}_{4}$ and the coset $\mathcal{S}_{4} / \mathcal{S}_{3}$.

\section{THE MODEL, SYMMETRY, AND VACUA}

\section{A. The model}

We study a $U(1)$ gauge theory with $N_{F}$ charged complex scalar fields $H^{A}\left(A=1,2, \ldots, N_{F}\right)$ and $N_{F}^{\prime}$ real scalar fields $\Sigma^{A^{\prime}}\left(A^{\prime}=1,2, \ldots, N_{F}^{\prime}\right)$ in $D+1$-dimensional spacetime. The Lagrangian is given by

$$
\begin{aligned}
\mathcal{L}= & -\frac{1}{4 e^{2}} F_{\mu \nu} F^{\mu \nu}+\frac{1}{2 e^{2}} \sum_{A^{\prime}=1}^{N_{F}^{\prime}} \partial_{\mu} \Sigma^{A^{\prime}} \partial^{\mu} \Sigma^{A^{\prime}}+D_{\mu} H\left(D^{\mu} H\right)^{\dagger} \\
& -\frac{1}{2 e^{2}} Y^{2}-\sum_{A^{\prime}=1}^{N_{F}^{\prime}}\left(\Sigma^{A^{\prime}} H-H M^{A^{\prime}}\right)\left(\Sigma^{A^{\prime}} H-H M^{A^{\prime}}\right)^{\dagger},
\end{aligned}
$$

where $H$ is an $N_{F}$ component row vector made of $H^{A}$,

$$
H=\left(H^{1}, H^{2}, \ldots, H^{N_{F}}\right),
$$

$Y$ is a scalar quantity defined by

$$
Y=e^{2}\left(v^{2}-H H^{\dagger}\right),
$$

and $M^{A^{\prime}}\left(A^{\prime}=1, \ldots, N_{F}^{\prime}\right)$ are $N_{F}$ by $N_{F}$ real diagonal mass matrices defined by

$$
M^{A^{\prime}}=\operatorname{diag}\left(m_{A^{\prime}, 1}, m_{A^{\prime}, 2}, \ldots, m_{A^{\prime}, N_{F}}\right) .
$$

The spacetime index $\mu$ runs from 0 to $D$, and $F_{\mu \nu}$ is a $U(1)$ gauge field strength. The coupling constants in the Lagrangian in Eq. (2.2) are taken to be the so-called Bogomol'nyi limit. ${ }^{2}$

Let us discuss the symmetry structure of our model. When all the mass matrices are proportional to the unit matrix, the flavor symmetry for $H$ is $S U\left(N_{F}\right)$. It reduces to

\footnotetext{
${ }^{2}$ This does not immediately imply that the model can be made supersymmetric by adding fermions. Only in certain cases, this can be supersymmetric.
}

a subgroup according to degeneracy of the mass eigenvalues. Let $\boldsymbol{m}_{A}$ be an $N_{F}^{\prime}$ vector whose components are the $A$ th diagonal elements of $M^{A^{\prime}}$ s, namely,

$$
\boldsymbol{m}_{A}=\left(m_{1, A}, m_{2, A}, \ldots, m_{N_{F}^{\prime}, A}\right) \text {. }
$$

Then, the flavor symmetry $S U\left(N_{F}\right)$ is explicitly broken maximally to $U(1)^{N_{\mathrm{F}}-1}$ when

$$
\boldsymbol{m}_{A} \neq \boldsymbol{m}_{B}, \quad \text { if } A \neq B
$$

When all the mass matrices are zero, there is a flavor symmetry $O\left(N_{F}^{\prime}\right)$ acting on $\boldsymbol{\Sigma}=\left(\Sigma^{1}, \Sigma^{2}, \ldots, \Sigma^{N_{F}^{\prime}}\right)$.

When the masses are specially tuned, a discrete symmetry appears. This can be found by studying the following term in the potential:

$$
\sum_{A^{\prime}} \Sigma^{A^{\prime}} H M^{A^{\prime}} H^{\dagger}=\sum_{A^{\prime}, A} \Sigma^{A^{\prime}} m_{A^{\prime}, A}\left|H^{A}\right|^{2}=\Sigma^{T} \mathcal{M}|\vec{H}|^{2},
$$

where $\mathcal{M}$ is an $N_{F}^{\prime}$ by $N_{F}$ matrix defined by $(\mathcal{M})_{A^{\prime}, A}=m_{A^{\prime}, A}$, and $\overrightarrow{|H|^{2}}$ is an $N_{F}$ vector defined by $\overrightarrow{|H|^{2}}=\left(\left|H^{1}\right|^{2},\left|H^{2}\right|^{2}, \cdots,\left|H^{N_{F}}\right|^{2}\right)$. Thus, a transformation $\boldsymbol{\Sigma} \rightarrow U_{\Sigma} \boldsymbol{\Sigma}, \overrightarrow{|H|^{2}} \rightarrow U_{H} \overrightarrow{|H|^{2}}$ satisfying

$$
U_{\Sigma}^{T} \mathcal{M} U_{H}=\mathcal{M}
$$

is an extra symmetry of $\mathcal{L}$.

To illustrate such a discrete symmetry, let us give a concrete example for the case of $N_{F}=3$ and $N_{F}^{\prime}=2$ with

$$
\begin{aligned}
& M^{A^{\prime}=1}=m \operatorname{diag}\left(1, \cos \frac{2 \pi}{3}, \cos \frac{4 \pi}{3}\right), \\
& M^{A^{\prime}=2}=m \operatorname{diag}\left(0, \sin \frac{2 \pi}{3}, \sin \frac{4 \pi}{3}\right),
\end{aligned}
$$

which give

$$
\begin{aligned}
& \boldsymbol{m}_{1}=m(1,0), \quad \boldsymbol{m}_{2}=m\left(\cos \frac{2 \pi}{3}, \sin \frac{2 \pi}{3}\right), \\
& \boldsymbol{m}_{3}=m\left(\cos \frac{4 \pi}{3}, \sin \frac{4 \pi}{3}\right)
\end{aligned}
$$

This can equally be expressed in the matrix form as

$$
\mathcal{M}=m\left(\begin{array}{ccc}
1 & \cos \frac{2 \pi}{3} & \cos \frac{4 \pi}{3} \\
0 & \sin \frac{2 \pi}{3} & \sin \frac{4 \pi}{3}
\end{array}\right) .
$$

One can easily check that $\mathcal{M}$ is invariant under a set of the following transformations: 


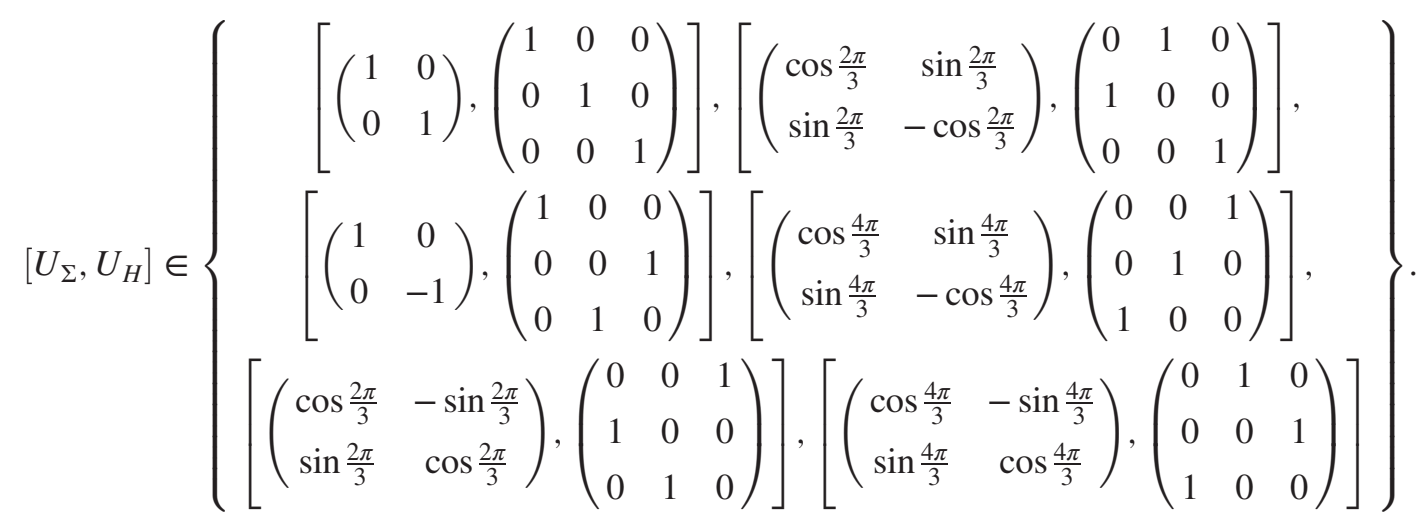

It is obvious that $\left\{U_{H}\right\}$ is the complete set of the symmetric group of degree three $\mathcal{S}_{3}$, which is the group of all permutations of a three-element set. Moreover, $\left\{U_{\Sigma}\right\}$ is the 2 by 2 matrix representation of $\mathcal{S}_{3}$. We should verify if the other terms in the Lagrangian is invariant or not. Clearly, both $H H^{\dagger}$ and $D_{\mu} H\left(D^{\mu} H\right)^{\dagger}$ are invariant under any transformations $\left\{U_{H}\right\}$. Then, $\sum_{A^{\prime}} H M_{A^{\prime}}{ }^{2} H^{\dagger}$ is the only term which we need to check. For the special mass matrix given in Eq. (2.9), we have

$$
\sum_{A^{\prime}=1}^{2} H M_{A^{\prime}}{ }^{2} H^{\dagger}=m^{2} H H^{\dagger},
$$

and so it is also invariant. Henceforth, the global symmetry of the Lagrangian is $U(1)^{2} \times \mathcal{S}_{3}$ in this special case. ${ }^{3}$ This kind of discrete symmetry will play an important role when we construct an exact solution of a domain wall junction.

\section{B. Vacua}

Since the scalar potential is positive semidefinite, a classical vacuum of the theory is determined by $V=0$. Thus, the vacuum condition reads

$$
H H^{\dagger}=v^{2}, \quad \Sigma^{A^{\prime}} H-H M^{A^{\prime}}=0 .
$$

In general, there are $N_{F}$ discrete vacua given by

$$
\langle A\rangle: H^{B}=v \delta_{A}^{B}, \quad \Sigma^{B^{\prime}}=m_{B^{\prime}, A} .
$$

While we can specify the vacua by using either $H^{A}$ or $\Sigma^{A^{\prime}}$, it will turn out that $\Sigma^{A^{\prime}}$ is more useful and so we express the vacua in the $N_{F}^{\prime}$ dimensional internal space spanned by $\Sigma^{A^{\prime}}$. The vacua are identical to the discrete points in the $\Sigma$ space. The $\langle A\rangle$ vacuum corresponds to the point specified by

$$
\langle A\rangle: \mathbf{\Sigma}=\boldsymbol{m}_{A},
$$

\footnotetext{
${ }^{3}$ In Ref. [80], the symmetry is said as $\mathbb{Z}_{3}$ but it is indeed $\mathcal{S}_{3}$.
}

where $\boldsymbol{m}_{A}$ is the $N_{F}^{\prime}$ vector whose components are the $A$ th eigenvalues of $M^{A^{\prime}}$, namely, $\boldsymbol{m}_{A}=\left(m_{1, A}, m_{2, A}, \ldots, m_{N_{F}^{\prime}, A}\right)$. Hence, the number of the discrete vacua depends only on $N_{F}$. Figure 1 shows three examples with $N_{F}=3$ and $N_{F}^{\prime}=1,2,3$. Note that the vacua have an $N_{F}^{\prime}$ dimensional structure when $N_{F} \geq N_{F}^{\prime}$.

\section{Comments on supersymmetry}

Note that the parameters of the above Lagrangian are tuned in such a way that it becomes identical to a bosonic part of supersymmetric Lagrangian. For comparison, let us write down the $\mathcal{N}=2$ supersymmetric Lagrangian in $3+$ 1 dimensions,

$$
\begin{aligned}
\mathcal{L}_{\mathcal{N}=2}^{(D=3)}= & -\frac{1}{4 e^{2}} F_{\mu \nu} F^{\mu \nu}+\frac{1}{2 e^{2}} \sum_{A^{\prime}=1}^{2} \partial_{\mu} \Sigma^{A^{\prime}} \partial^{\mu} \Sigma^{A^{\prime}} \\
& +\sum_{i=1}^{2} D_{\mu} H_{i}\left(D^{\mu} H_{i}\right)^{\dagger}-\frac{1}{2 e^{2}} \sum_{a=1}^{3} Y_{a}^{2} \\
& -\sum_{A^{\prime}=1}^{2} \sum_{i=1}^{2}\left(\Sigma^{A^{\prime}} H_{i}-H_{i} M^{A^{\prime}}\right)\left(\Sigma^{A^{\prime}} H_{i}-H_{i} M^{A^{\prime}}\right)^{\dagger},
\end{aligned}
$$

with

$$
Y_{a}=e^{2}\left(c_{a}-\vec{H} \sigma_{a} \vec{H}^{\dagger}\right), \quad \vec{H}=\left(H_{1}, H_{2}\right) .
$$

This is nothing but the $\mathcal{N}=2$ SQED with $A_{\mu}$ and $\Sigma^{1,2}$ being bosonic components of a vector multiplet whereas $H_{i=1,2}$ being those of hypermultiplets (the subscription $i$ is the index of the $S U(2)_{R}$ symmetry). $Y_{a=1,2}$ are the so-called Fayet-Illiopoulos (FI) $F$ terms, and $Y_{a=3}$ is called the FI $D$ term, which forms an $S U(2)_{R}$ triplet. The constants $c_{a}$ are the FI terms which we can set $c_{a}=\left(0,0, v^{2}\right)$ without loss of generality.

Now, the SUSY vacua read very similar to those in Eq. (2.15) as

$$
\langle A\rangle: H_{1}^{B}=v \delta_{A}^{B}, \quad H_{2}^{B}=0, \quad \Sigma^{B^{\prime}}=m_{B^{\prime}, A} .
$$




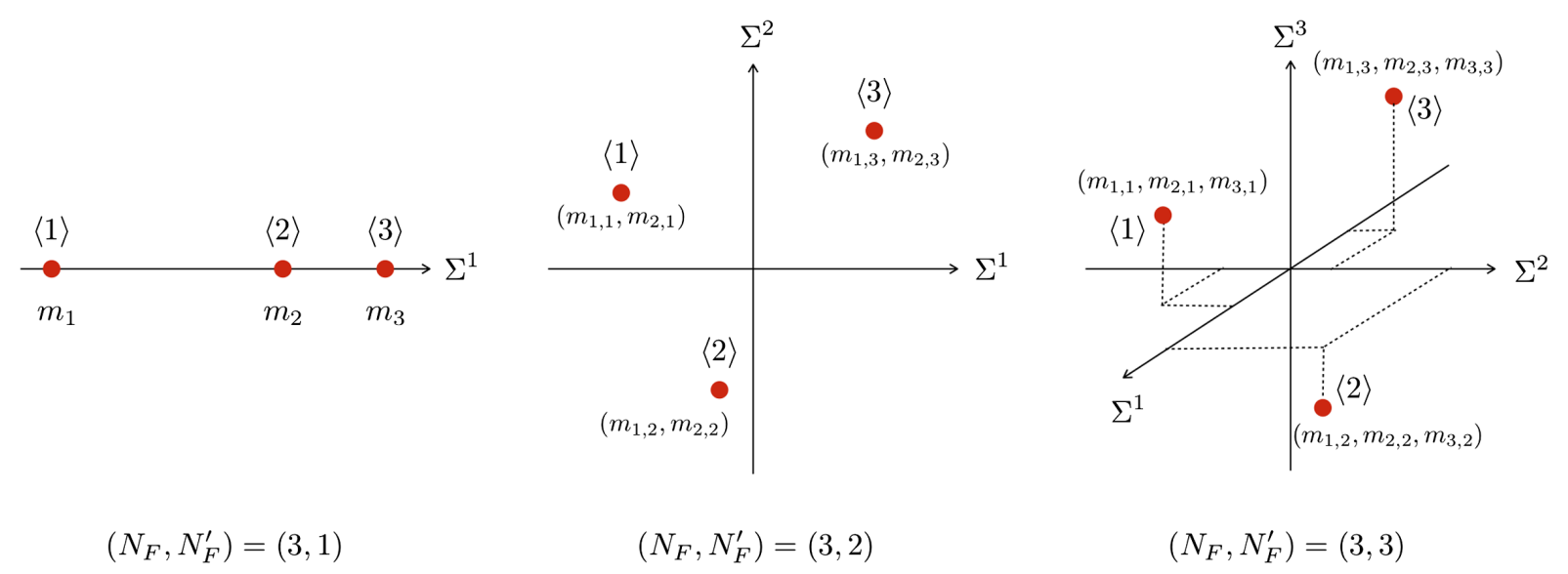

FIG. 1. The discrete vacua correspond to isolated points (red circles) in the internal $\boldsymbol{\Sigma}$ space. We show three examples with $N_{F}=3$ and $N_{F}^{\prime}=1,2,3$.

One can easily be convinced that $\mathcal{L}$ with $D=3$ and $N_{F}^{\prime}=2$ is identical to $\mathcal{L}_{\mathcal{N}=2}^{(D=3)}$ except for the additional complex scalar $H_{2}$. However, it was found that $H_{2}$ is completely inert for the BPS states which we are interested in this paper. Therefore, the Lagrangian (2.2) with ignoring the sterile scalar $\mathrm{H}_{2}$ makes sense [we can include $\mathrm{H}_{2}$ in Eq. (2.2) but it will be identically zero for BPS configurations].

\section{BPS EQUATIONS FOR DOMAIN WALLL JUNCTIONS}

From now on, we will investigate BPS states of $\mathcal{L}$ in Eq. (2.2) with $N_{F}^{\prime}=D$ under the expectation that the BPS states can exist only when the symmetric structures in the spatial and the internal spaces are identical as mentioned in the Introduction. In what follows, the Roman index $m$ stands for the spacial index as $m=1,2, \ldots, D$ and we will use it for the index of $N_{F}^{\prime}\left(m \equiv A^{\prime}\right)$. The energy density of static configurations is

$$
\begin{aligned}
\mathcal{E}= & \frac{1}{2 e^{2}} \sum_{m>n} F_{m n}^{2}+\frac{1}{2 e^{2}} \sum_{m, n}\left(\partial_{m} \Sigma_{n}\right)^{2}+\sum_{m} D_{m} H\left(D_{m} H\right)^{\dagger} \\
& +\frac{1}{2 e^{2}} Y^{2}+\sum_{m}\left(\Sigma_{m} H-H M_{m}\right)\left(\Sigma_{m} H-H M_{m}\right)^{\dagger} .
\end{aligned}
$$

In order to perform a standard Bogomol'nyi completion to this energy density, let us first note that the derivative terms of $\Sigma_{m}$ can be cast into the following form:

$$
\begin{aligned}
\sum_{m, n}\left(\partial_{m} \Sigma_{n}\right)^{2}= & \sum_{m}\left(\partial_{m} \Sigma_{m}\right)^{2}+\sum_{m>n}\left\{\left(\partial_{m} \Sigma_{n}\right)^{2}+\left(\partial_{n} \Sigma_{m}\right)^{2}\right\} \\
= & \left(\sum_{m} \xi_{m} \partial_{m} \Sigma_{m}\right)^{2}+\sum_{m>n}\left(\partial_{m} \Sigma_{n}+\chi_{m n} \partial_{n} \Sigma_{m}\right)^{2} \\
& -2 \sum_{m>n}\left(\xi_{m} \xi_{n} \partial_{m} \Sigma_{m} \partial_{n} \Sigma_{n}+\chi_{m n} \partial_{m} \Sigma_{n} \partial_{n} \Sigma_{m}\right),
\end{aligned}
$$

where we have introduced the signs $\chi_{m n}, \xi_{m}= \pm 1$. Then, the Bogomol'nyi completion goes as follows:

$$
\begin{aligned}
\mathcal{E}= & \frac{1}{2 e^{2}} \sum_{m>n}\left\{F_{m n}^{2}+\left(\partial_{m} \Sigma_{n}+\chi_{m n} \partial_{n} \Sigma_{m}\right)^{2}\right\} \\
& +\frac{1}{2 e^{2}}\left(\sum_{m} \xi_{m} \partial_{m} \Sigma_{m}-Y\right)^{2} \\
& +\sum_{m}\left\{D_{m} H+\xi_{m}\left(\Sigma_{m} H-H M_{m}\right)\right\} \\
& \times\left\{D_{m} H+\xi_{m}\left(\Sigma_{m} H-H M_{m}\right)\right\}^{\dagger} \\
& +\sum_{m} \xi_{m} \mathcal{Z}_{m}+\sum_{m>n} \xi_{m} \xi_{n} \mathcal{Y}_{m n}+\sum_{m} \partial_{m} \mathcal{J}_{m},
\end{aligned}
$$

where we have introduced $\mathcal{Z}_{m}, \mathcal{Y}_{m n}$, and $\mathcal{J}_{m}$ as

$$
\begin{gathered}
\mathcal{Z}_{m}=v^{2} \partial_{m} \Sigma_{m}, \\
\mathcal{Y}_{m n}=-\frac{1}{e^{2}}\left(\partial_{m} \Sigma_{m} \partial_{n} \Sigma_{n}+\chi_{m n} \xi_{m} \xi_{n} \partial_{m} \Sigma_{n} \partial_{n} \Sigma_{m}\right), \\
\mathcal{J}_{m}=-\xi_{m}\left(\Sigma_{m} H-H M_{m}\right) H^{\dagger} .
\end{gathered}
$$

The terms $\mathcal{Z}_{m}$ and $\mathcal{Y}_{m n}$ can contribute only topologically. Indeed, the first quantity $\mathcal{Z}_{m}$ is related to a domain wall tension measured along the $x^{m}$ direction as

$Z_{m}=\int_{-\infty}^{\infty} d x^{m} \xi_{m} \mathcal{Z}_{m}=v^{2} \xi_{m}\left(\left.\Sigma_{m}\right|_{x^{m}=+\infty}-\left.\Sigma_{m}\right|_{x^{m}=-\infty}\right)$.

It is well known that $Z_{m}$ is always positive regardless of the choice of $\xi_{m}$, which is naturally understood as the domain wall tension. On the other hand, the contribution of $\mathcal{J}_{m}$ vanishes since it is asymptotically zero because of the vacuum condition (2.14). The term $\mathcal{Y}_{m n}$ is not topological as it is in general. To make it topological, we need to impose an additional condition 


$$
\chi_{m n} \xi_{m} \xi_{n}=-1
$$

Then, it becomes

$$
\begin{aligned}
\mathcal{Y}_{m n} & =-\frac{1}{e^{2}}\left(\partial_{m} \Sigma_{m} \partial_{n} \Sigma_{n}-\partial_{m} \Sigma_{n} \partial_{n} \Sigma_{m}\right) \\
& =-\frac{1}{e^{2}} \operatorname{det}\left(\begin{array}{ll}
\partial_{m} \Sigma_{m} & \partial_{m} \Sigma_{n} \\
\partial_{n} \Sigma_{m} & \partial_{n} \Sigma_{n}
\end{array}\right),
\end{aligned}
$$

and its integration over the $x^{m}-x^{n}$ plane gives another topological quantity, ${ }^{4}$

$$
Y_{m n}=\xi_{m} \xi_{n} \int d x^{m} d x^{n} \mathcal{Y}_{m n}=-\frac{\xi_{m} \xi_{n}}{e^{2}} S_{m n},
$$

where we have defined

$$
S_{m n} \equiv \int d x^{m} d x^{n} \operatorname{det}\left(\begin{array}{ll}
\partial_{m} \Sigma_{m} & \partial_{m} \Sigma_{n} \\
\partial_{n} \Sigma_{m} & \partial_{n} \Sigma_{n}
\end{array}\right)
$$

The term $S_{m n}$ corresponds to an area of the region in the $\Sigma^{m}-\Sigma^{n}$ plane mapped from the whole $x^{m}-x^{n}$ plane by the function $\left(\Sigma_{m}\left(x^{m}, x^{n}\right), \Sigma_{n}\left(x^{m}, x^{n}\right)\right)$ with all other coordinates $x^{k}(k \neq m, n)$ being fixed. Thus, with appropriately normalized, the $S_{m n}$ represents the degree of the map counting how many times a certain area defined below in the $\Sigma_{m}-\Sigma_{n}$ plane is covered when the real $x_{m}-x_{n}$ plane is swept once. Precisely speaking, $S_{m n}$ can be positive or negative, and its absolute value is the area. Interestingly, $\xi_{m} \xi_{n} Y_{m n}$ is always negative $e^{5}$ independent of choice of the signs $\xi_{m}$ and $\xi_{n}$, so it should be understood as a sort of binding energy among domain walls [73-75].

Once we have confirmed that all the terms in the third line of Eq. (3.3) are topological, it assures us that the energy density is bounded from below as

$$
\mathcal{E} \geq \sum_{m} \xi_{m} \mathcal{Z}_{m}+\sum_{m>n} \xi_{m} \xi_{n} \mathcal{Y}_{m n}+\sum_{m} \partial_{m} \mathcal{J}_{m}
$$

This is saturated when the following first order equations are satisfied:

$$
\begin{gathered}
F_{m n}=0, \\
\xi_{m} \partial_{m} \Sigma_{n}-\xi_{n} \partial_{n} \Sigma_{m}=0, \\
\sum_{m} \xi_{m} \partial_{m} \Sigma_{m}-Y=0,
\end{gathered}
$$

\footnotetext{
${ }^{4}$ Note that $\mathcal{Y}_{m n}(m>n)$ can be cast into a total derivative form in the $x^{m}-x^{n}$ plane as $\mathcal{Y}_{m n}=-\frac{1}{e^{2}} \partial_{k}\left(\epsilon^{k l} \Sigma_{m} \partial_{l} \Sigma_{n}\right)$ where $k$ and $l$ take the value in $m$ and $n$, and $\epsilon^{m n}=-\epsilon^{n m}=1$. Therefore, this is a topological charge density.

${ }^{5}$ The negativeness of $Y_{m n}$ was proved for the $D=2$ case, namely, $m=1$ and $n=2$, in Ref. [81]. The same proof holds for the generic $Y_{m n}$ in $D \geq 2$, so we do not repeat it here.
}

$$
\xi_{m} D_{m} H+\left(\Sigma_{m} H-H M_{m}\right)=0,
$$

where $m, n=1,2, \ldots, D$. One can verify that all solutions of the above BPS equations solve the full equations of motion. ${ }^{6}$ The stability of the solutions is ensured by the fact that they saturate the Bogomol'nyi energy bound. These are a set of the BPS equations of the domain wall junction in $D$ dimensions $(D \geq 3)$ obtained for the first time. Note that they are a $D$-dimensional generalization of the BPS equations of the domain wall junction in $D=2$ cases studied in Refs. [80,81]. Note that it is called the $\frac{1}{4}$ BPS equations when $D=2$. It is because a solution of the equations preserves a quarter of the supersymmetry when embedded into a supersymmetric theory Refs. [59-61]. Although we have not dealt with any supersymmetry at all in this paper, one can grasp the origin of $\frac{1}{4}$ BPS-ness by looking at our BPS equations. ${ }^{7}$ When $D=2$, we have two signs $\xi_{1,2}= \pm 1$. Therefore, depending on $\xi_{1,2}$, there exist four different ways for performing the Bogomol'nyi completion to obtain BPS equations. Turning to our BPS equations in $D$ dimensions, they involve the $D$ signs $\xi_{1,2, \ldots, D}$. Therefore, there exist $2^{D}$ different ways to perform the Bogomol'nyi completion of the energy density.

\section{A PLANAR $\mathcal{S}_{3} / \mathcal{S}_{2}$ DOMAIN WALL JUNCTION IN $D=2$ : A REVIEW}

As worming up, we briefly review an exact solution for a $\mathcal{S}_{3} / \mathcal{S}_{2}$ domain wall junction in the case with $N_{F}=3$ and $D=N_{F}^{\prime}=2$, which was found in Ref. [80] (with $N_{F}=$ $D=3$ and $\left.N_{F}^{\prime}=2\right) .{ }^{8}$ Now, the indices $m, n$ run from 1 to 2. The mass matrices are those given in Eqs. (2.9) and (2.10), leading to the $\mathcal{S}_{3}$ symmetric group. Following the generic argument obtained in Eq. (2.15), we find three discrete vacua shown in Fig. 2(a). In the first vacuum $\langle 1\rangle: \boldsymbol{\Sigma}=\boldsymbol{m}_{1}$, the discrete symmetry is spontaneously broken as $\mathcal{S}_{3} \rightarrow \mathcal{S}_{2}$, where the elements of the unbroken $\mathcal{S}_{2}$ symmetry are given by $U_{\Sigma} \in\left\{\left(\begin{array}{ll}1 & 0 \\ 0 & 1\end{array}\right),\left(\begin{array}{cc}1 & 0 \\ 0 & -1\end{array}\right)\right\}$, and the corresponding $U_{H}$ elements are

$$
U_{H} \in\left\{\left(\begin{array}{lll}
1 & 0 & 0 \\
0 & 1 & 0 \\
0 & 0 & 1
\end{array}\right),\left(\begin{array}{lll}
1 & 0 & 0 \\
0 & 0 & 1 \\
0 & 1 & 0
\end{array}\right)\right\} .
$$

\footnotetext{
${ }^{6}$ Note that if we took the wrong $\operatorname{sign} \chi_{m n} \xi_{m} \xi_{n}=+1$ instead of Eq. (3.8), the BPS equations conflict with equations of motion.

Indeed, the $\frac{1}{4}$ BPS equations can also be derived via appropriate supersymmetry preserving conditions to the supersymmetry transformation on the fermionic fields. The sings $\xi_{1,2}$ enter in this argument when we select $\frac{1}{4}$ of the supercharges by two appropriate projection operators.

${ }^{8}$ It was called the $\mathbb{Z}_{3}$ junction in Ref. [80]. However, note that the symmetry of the Lagrangian is $\mathcal{S}_{3}$ but not $\mathbb{Z}_{3}$ and that we call it the $\mathcal{S}_{3} / \mathcal{S}_{2}$ domain wall junction.
} 


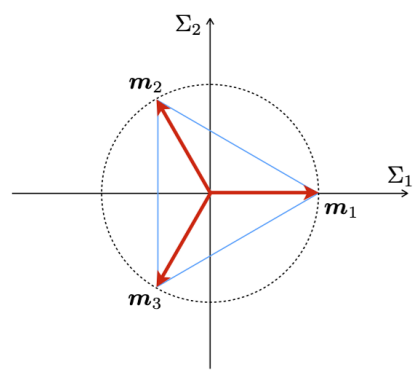

(a) $\Sigma_{1}-\Sigma_{2}$ plane

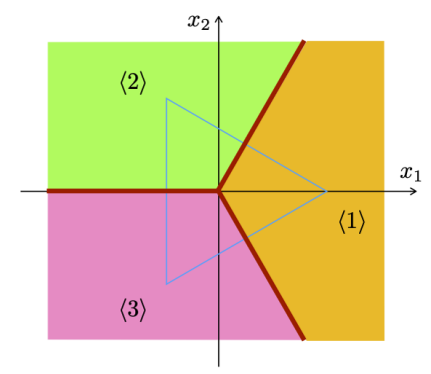

(b) $x_{1}-x_{2}$ plane

FIG. 2. (a) The $\mathcal{S}_{3} / \mathcal{S}_{2}$ mass vectors in the $\Sigma_{1}-\Sigma_{2}$ plane. (b) The dual picture depicted in the $x_{1}-x_{2}$ plane.

Thus, the vacuum structure respects the symmetry breaking pattern as $\mathcal{S}_{3} / \mathcal{S}_{2}$ which corresponds to the three vertices of the equilateral triangle.

To be concrete, we will set $\xi_{1}=\xi_{2}=+1$ without loss of generality in what follows. The gauge field plays no role in solutions, and so they vanish $A_{0}=A_{1}=A_{2}=0$. Then, Eq. (3.13) is trivially satisfied. Equation (3.14) is solved by introducing a scalar potential $\phi\left(x^{1}, x^{2}\right)$ for $\Sigma_{1,2}$ by

$$
\Sigma_{m}=\partial_{m} \phi, \quad(m=1,2)
$$

Then, one can verify that Eq. (3.16) is solved by

$$
H=v e^{-\phi}\left(e^{m_{1} \cdot x}, e^{m_{2} \cdot x}, e^{m_{3} \cdot x}\right)
$$

where the mass vectors are those given in Eq. (2.10) and $\boldsymbol{x}=\left(x_{1}, x_{2}\right)$. Note that we have omitted translational moduli parameters since they can always be absorbed by the translational invariance. ${ }^{9}$ Finally, we are left with Eq. (3.15) which is now expressed in terms of $\phi$ as

$$
\frac{1}{e^{2} v^{2}} \triangle_{2} \phi=1-e^{-2 \phi} \psi
$$

where $\triangle_{2}$ stands for the two-dimensional Laplacian, and we have introduced a semipositive function

$$
\psi \equiv e^{2 m_{1} \cdot x}+e^{2 m_{2} \cdot x}+e^{2 m_{3} \cdot x}
$$

No exact solutions for this equation have been known so far, except for the special case [80] in which the model parameters are tuned as

\footnotetext{
${ }^{9}$ Although the domain wall junction consists of the three domain walls, the number of the independent moduli is not three but two. This is because, once we fix the positions of the two domain walls, the position of the third one is automatically determined. Therefore, we can always fix the moduli by the twodimensional translations without loss of generality. See Ref. [81] for more details.
}

$$
e v=\sqrt{\frac{3}{2}} m
$$

In this special case, an exact solution of Eq. (4.3) is given by

$$
\phi=\log \left(e^{\boldsymbol{m}_{1} \cdot \boldsymbol{x}}+e^{\boldsymbol{m}_{2} \cdot \boldsymbol{x}}+e^{\boldsymbol{m}_{3} \cdot \boldsymbol{x}}\right) .
$$

This corresponds to the exact solution found in Ref. [80].

Note that the previous work [80] for $D=2$ solved the BPS equations in a quite different way where the scalar potential was not introduced. The procedure of obtaining the solution presented here is peculiar to the present work. We would like to stress that introducing the scalar potential $\phi$ makes things transparent and is an important clue for obtaining the exact solutions in higher dimensions.

As a consistency check, let us verify the contribution of $\mathcal{Y}_{12}$ to the energy density. It can be expressed as

$$
\begin{aligned}
\mathcal{E} \supset \xi_{1} \xi_{2} \mathcal{Y}_{12} & =-\frac{1}{e^{2}}\left[\partial_{1}^{2} \phi \partial_{2}^{2} \phi-\left(\partial_{1} \partial_{2} \phi\right)^{2}\right] \\
& =-\frac{27 m^{4}}{4 e^{2}} e^{-3 \phi}<0 .
\end{aligned}
$$

Thus, it is negative whole over the $x^{1}-x^{2}$ plane, and so that its integration is also negative as expected. Figure 3 shows several plots for the exact solution.

It is worthwhile mentioning how useful depicting the solution on the $\Sigma_{1}-\Sigma_{2}$ plane is. We should emphasize that Fig. 2(a) has almost all information not only of the vacua but also of $1 / 2$ BPS domain walls and 1/4 BPS domain wall junctions [81]. As we explained, the three vertices of the equilateral triangle correspond to the three vacua $\langle A\rangle$ with $\boldsymbol{\Sigma}=\boldsymbol{m}_{A}(A=1,2,3)$. In addition, the edge vector $\boldsymbol{m}_{A}-\boldsymbol{m}_{B}$ is identical to the domain wall interpolating the vacua $\langle A\rangle$ and $\langle B\rangle$. The domain wall tension can easily be read from the length of the edge as $Z_{A B}=v^{2}\left|\boldsymbol{m}_{A}-\boldsymbol{m}_{B}\right|$ from Eq. (3.7). Finally, the junction of the three domain walls corresponds to the face of the triangle. As we have explained, the junction charge $Y_{12}$ is proportional to the area of the triangle, and so it can be easily calculated as $Y_{12}=-\frac{(3+\sqrt{3}) m^{2}}{e^{2}}$. This is not all information we can get from the $\Sigma_{1}-\Sigma_{2}$ plane, and we can also read the geometric information of the domain wall junction in the $x^{1}-x^{2}$ plane. Namely, we can find angles of the domain walls extending from the junction point: they are the orthonormal lines of the edges of the triangle. This can be more rigorously confirmed by regarding $\mathbf{\Sigma}\left(x_{1}, x_{2}\right)$ as a function which maps the real $x_{1}-x_{2}$ plane to the internal $\Sigma_{1}-\Sigma_{2}$ plane. Figure 4 shows the image of the mapping: the whole $x_{1}-x_{2}$ plane is mapped onto the compact equilateral triangle in the $\Sigma_{1}-\Sigma_{2}$ plane. As can be shown in Fig. 4(c), generic points are mapped onto either of three vertices as expected. Hence, the configuration in the real space [shown in Fig. 2(b)] is a dual picture of the internal plane 


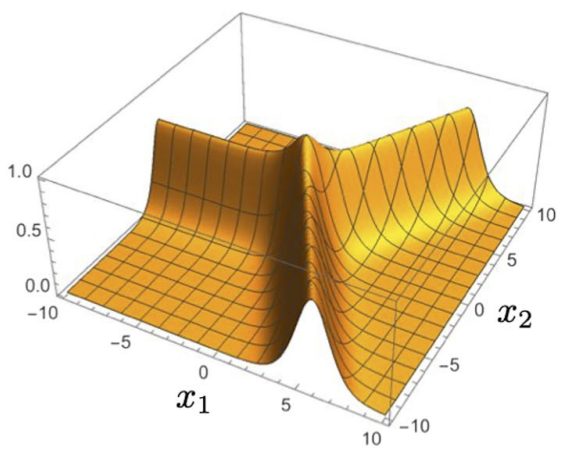

(a) $\mathcal{Z}_{1}+\mathcal{Z}_{2}$

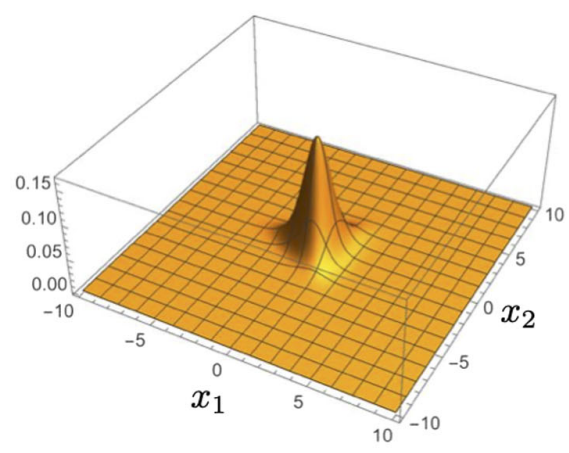

(b) $\left|\mathcal{Y}_{12}\right|$

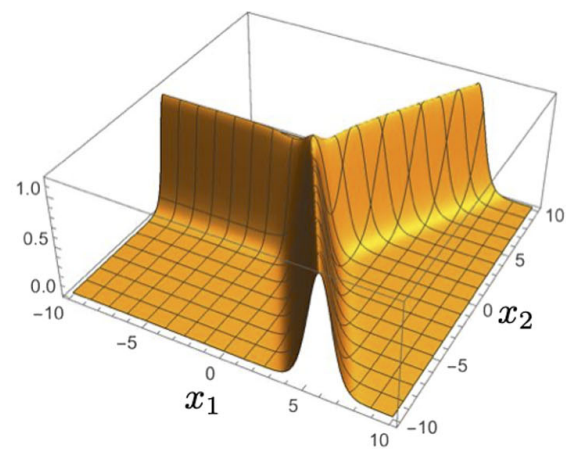

(c) total

FIG. 3. The exact solution of the $\mathcal{S}_{3} / \mathcal{S}_{2}$ planar domain wall junction. (a) The domain wall tension density $\mathcal{Z}_{1}+\mathcal{Z}_{2}$, (b) the absolute value of the $\mathcal{Y}_{12}$ charge density, and (c) the total energy density. We set $m=v=1$.

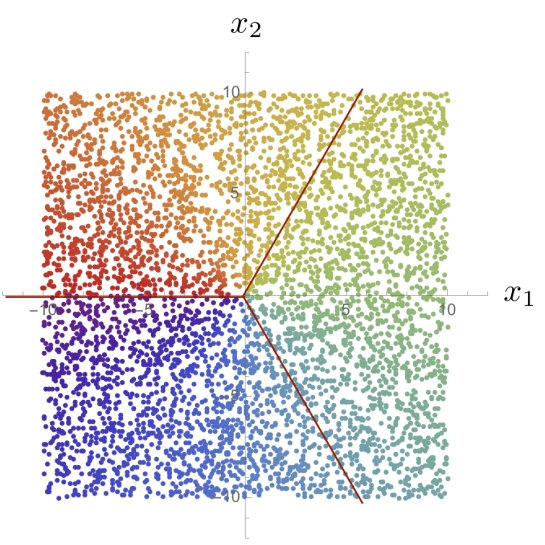

(a) $x_{1}-x_{2}$ plane

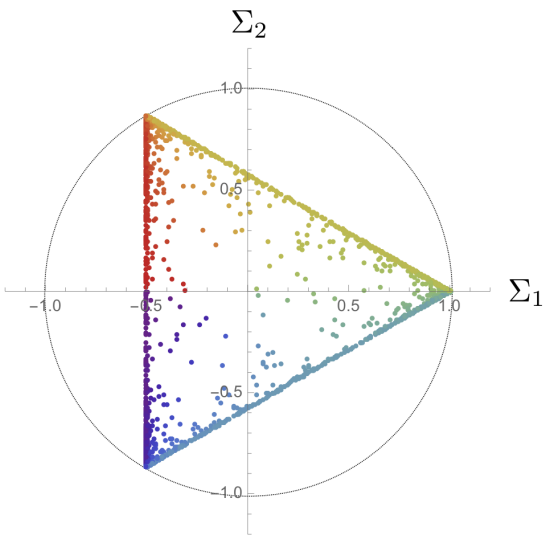

(b) $\Sigma_{1}-\Sigma_{2}$ plane

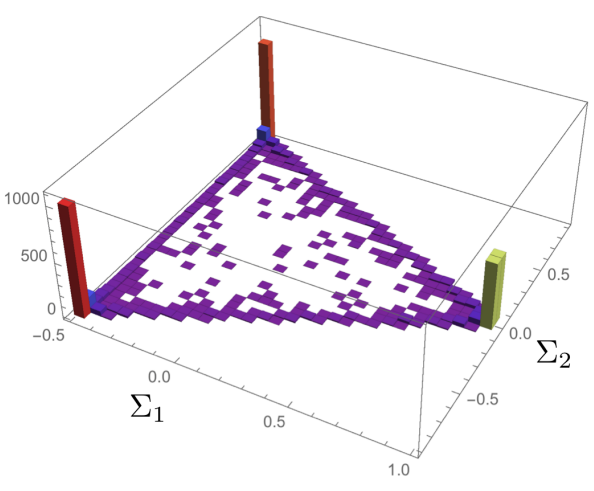

(c) histogram

FIG. 4. (a) 5000 points are randomly sampled in the region $x_{1,2} \in[-10,10]$, (b) the corresponding points in the $\Sigma_{1}-\Sigma_{2}$ plane are plot, and (c) the histogram counting the number of mapped points. The colors of points correspond to the angle in the $x_{1}-x_{2}$ plane.

[shown in Fig. 2(b)] [81]. They both have the almost same information, and neither of them has a big advantage in the $D=2$ case. However, as we will see below, the representation in the internal space is easier than the one in the real space when we go to higher dimensions $D \geq 3$. This is because that the former treats compact objects while the latter deals with noncompact configurations.

\section{A TETRAHEDRAL $\mathcal{S}_{4} / \mathcal{S}_{3}$ DOMAIN WALL JUNCTION IN $D=3$}

In this section, we construct a novel exact solution of a nonplanar 3D domain wall junction. To this end, we set $D=N_{F}^{\prime}=3(m=1,2,3)$ and $N_{F}=4(A=1,2,3,4)$. To be concrete, we take $\xi_{1}=\xi_{2}=\xi_{3}=+1$ in what follows.

In order to construct an exact solution, we need to arrange the model parameters in such a way that it has the highest discrete symmetry. Hence, our choice of the mass matrices is of the form

$$
\begin{aligned}
& M_{1}=\frac{m}{\sqrt{3}} \operatorname{diag}(1,-1,-1,1), \\
& M_{2}=\frac{m}{\sqrt{3}} \operatorname{diag}(-1,1,-1,1), \\
& M_{3}=\frac{m}{\sqrt{3}} \operatorname{diag}(-1,-1,1,1),
\end{aligned}
$$

or equivalently we have

$$
\begin{aligned}
& \boldsymbol{m}_{1}=\frac{m}{\sqrt{3}}(1,-1,-1), \\
& \boldsymbol{m}_{2}=\frac{m}{\sqrt{3}}(-1,1,-1), \\
& \boldsymbol{m}_{3}=\frac{m}{\sqrt{3}}(-1,-1,1),
\end{aligned}
$$




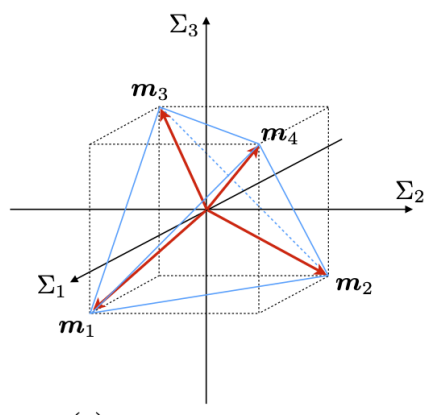

(a) internal $\Sigma_{1,2,3}$ space

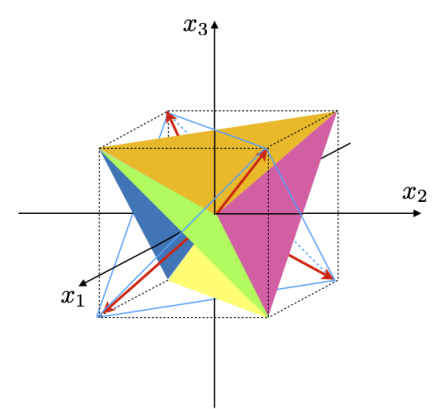

(b) real $x_{1,2,3}$ space

FIG. 5. (a) The regular tetrahedron in the $\boldsymbol{\Sigma}$ space. Each vertex corresponds to the vacuum. (b) The dual picture of (a) depicted in the real space. The painted triangles correspond to the domain walls. Only a part of the domain walls inside the auxiliary cube are shown.

$$
\boldsymbol{m}_{4}=\frac{m}{\sqrt{3}}(1,1,1)
$$

These are nothing but the four vertices of the regular tetrahedron which are inscribed by a sphere of radius $m$. Then, the discrete symmetry of the masses is the tetrahedral symmetry which is isomorphic to the symmetric group of degree four $\mathcal{S}_{4}$, as depicted in Fig. 5(a). So, we expect that the symmetry of the model is also $\mathcal{S}_{4}$. This can be verified by examining the rectangle mass matrix $\mathcal{M}$ defined in Eq. (2.7),

$$
\mathcal{M}=\frac{m}{\sqrt{3}}\left(\begin{array}{cccc}
1 & -1 & -1 & 1 \\
-1 & 1 & -1 & 1 \\
-1 & -1 & 1 & 1
\end{array}\right)
$$

This is indeed invariant under the transformation $\mathcal{M} \rightarrow$ $U_{\Sigma}^{T} \mathcal{M} U_{H}$ with any $4 \times 4$ matrices $U_{H}$ of $\mathcal{S}_{4}$ together with the corresponding $3 \times 3$ matrices $U_{\Sigma}$ which are explicitly shown in the Appendix. Furthermore, the mass matrices given in Eqs. (5.1)-(5.3) satisfy $M_{1}^{2}+M_{2}^{2}+M_{3}^{2}=m^{2} \mathbf{1}_{3}$, so that, similarly to Eq. (2.13), we have $\sum_{A^{\prime}=1}^{3} H M_{A^{\prime}}{ }^{2} H^{\dagger}=$ $m^{2} H H^{\dagger}$. Hence, all the terms in the Lagrangian are indeed $\mathcal{S}_{4}$ invariant.

Let us mention the vacua. There are four discrete vacua in the model, and selecting one among four correspond to choosing one vertex from the tetrahedron. We choose for instance the first vacuum $\langle 1\rangle: \Sigma=\boldsymbol{m}_{1}$. A subgroup $\mathcal{S}_{3}$ of $\mathcal{S}_{4}$ which transforms the remaining vertices $\boldsymbol{m}_{2,3,4}$ is unbroken. Hence, the spontaneous symmetry breaking $\mathcal{S}_{4} \rightarrow \mathcal{S}_{3}$ occurs in this case. The vacuum structure (the discrete four points) respects this and it is isomorphic to the coset $\mathcal{S}_{4} / \mathcal{S}_{3}$; see the Appendix for some details. This is a straightforward extension of $\mathcal{S}_{3} / \mathcal{S}_{2}$ in the previous subsection.

We are ready for constructing a novel exact solution of a nonplanar 3D domain wall junction in the $\mathcal{S}_{4}$ symmetric model. As before, all the gauge fields are set to be zero

$A_{\mu=0,1,2,3}=0$ so that Eq. (3.13) is trivially satisfied. Equation (3.14) can be solved by introducing an arbitrary scalar potential $\phi$ as

$$
\Sigma_{m}=\partial_{m} \phi, \quad(m=1,2,3) .
$$

Then, one can verify that Eq. (3.16) is solved by

$H^{A}=v e^{-\phi_{1}} w_{A}, \quad w_{A} \equiv e^{\boldsymbol{m}_{A} \cdot \boldsymbol{x}}, \quad(A=1,2,3,4)$.

Finally, we are left with Eq. (3.15) which is now expressed in terms of $\phi$ as

$$
\frac{1}{e^{2} v^{2}} \triangle_{3} \phi=1-e^{-2 \phi} \psi
$$

where $\triangle_{3}$ stands for the three-dimensional Laplacian, and we have introduced a semipositive function

$$
\psi \equiv \sum_{A=1}^{4} w_{A}^{2} .
$$

Thanks to the $\mathcal{S}_{4}$ symmetric masses given in Eqs. (5.4)(5.7), Eq. (5.11) can be exactly solved by

$$
\phi=\log \sum_{A=1}^{4} w_{A}
$$

only if the model parameters satisfy the following condition:

$$
e v=\frac{2}{\sqrt{3}} m
$$

This is the exact analytic solution of a nonplanar domain wall junction in $D=3$ obtained for the first time.

Now, we make a comment on the power of the scalar potential $\phi$. All the procedures to obtain the exact solutions for $D=3$ are straightforward extension of those for $D=2$ in the previous section. If we tried to solve the BPS equations without the aid of the scalar potential $\phi$, as was done for $D=2$ in Ref. [80], it was difficult to reach the BPS equations in $D=3$. $^{10}$ We thus have succeeded in obtaining the new exact solution in the case of $D=3$.

Similarly, to the planar $\mathcal{S}_{3} / \mathcal{S}_{2}$ junction in the previous section, we can compute the $\mathcal{Y}_{m n}$ charge $(m>n)$ explicitly showing negativeness,

\footnotetext{
${ }^{10}$ Furthermore, the discrete symmetry of the $D=2$ model was incorrectly understood as $\mathbb{Z}_{3}$ in Ref. [80]. We have found the correct symmetry $\mathcal{S}_{3}$ for $D=2$ and figured out that the correct extension of the discrete symmetry is $\mathcal{S}_{4}$ for $D=3$, which is also the important clue for discovery of the exact analytic solution in $D=3$.
} 
(a) vacua

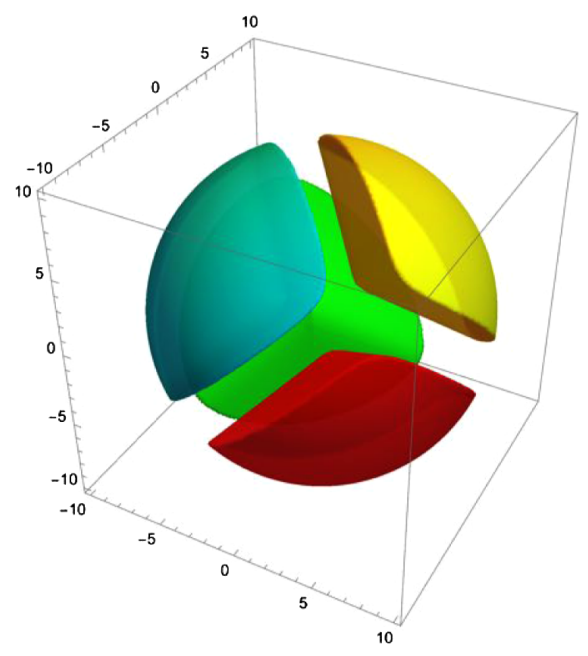

(a) $+(b)$

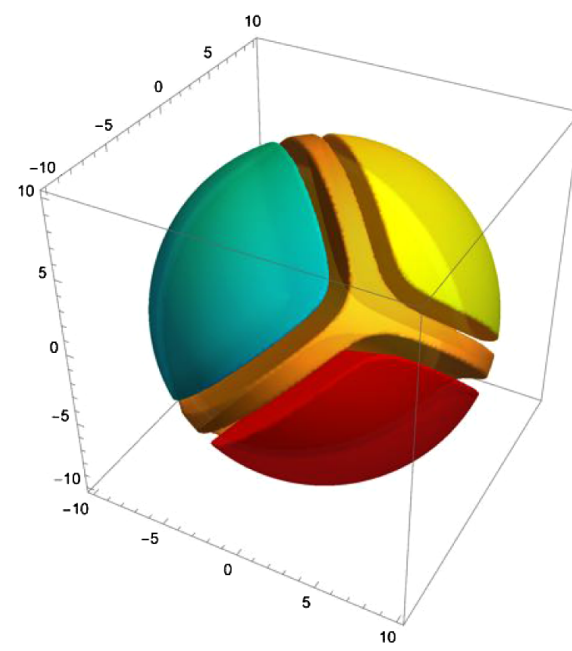

(b) domain walls

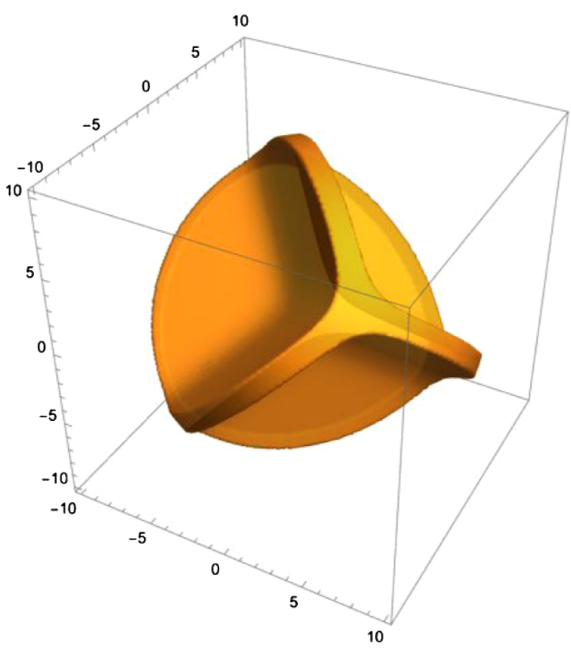

$(a)+(b)+(c)$

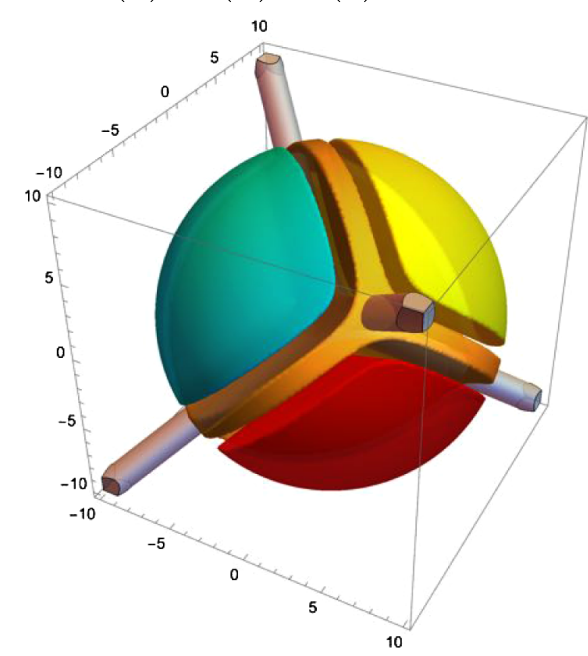

(c) DW junctions

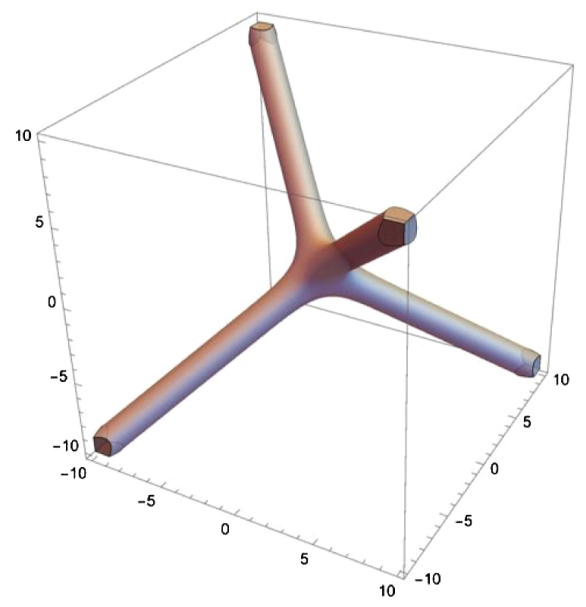

$(\mathrm{a})+(\mathrm{b})+(\mathrm{c})$

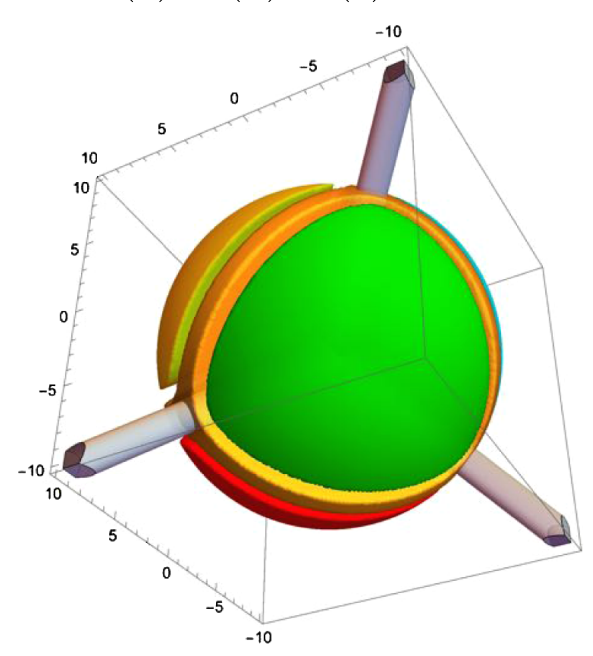

FIG. 6. The exact solution of the $\mathcal{S}_{4}$ tetrahedral domain wall junction. We set $m=v=1$. (a) shows the four domains of different vacua with $\{$ red, green, cyan, yellow $\}=\{\langle 1\rangle,\langle 2\rangle,\langle 3\rangle,\langle 4\rangle\}$, (b) shows the domain wall energy density $\sum_{m} \mathcal{Z}_{m}$, and (c) shows $\sum_{m>n} \mathcal{Y}_{m n}$ junction charge density. The panels in the second row are superpositions of the panels in the first row. The rightmost panel shows view from opposite side of the middle panel.

$$
\mathcal{Y}_{m n}=-\frac{16 m^{4}}{9 e^{2}} \frac{\sum_{C=1}^{4} w_{C}^{-1}}{\left(\sum_{C=1}^{4} w_{C}\right)^{3}}<0,
$$

for $m, n=1,2,3$.

In Fig. 6, we show several plots of the $\mathcal{S}_{4}$ symmetric exact solution in the real space. For ease of realization of the complicated 3D graphs, we individually plot the vacua, domain walls, and domain wall junctions in Figs. 6(a)-6(c), respectively. The red region of Fig. 6(a) is identified as the region of $\left\{\Sigma_{1}>\frac{m}{2}\right\} \wedge\left\{\Sigma_{2}<-\frac{m}{2}\right\} \wedge\left\{\Sigma_{3}<-\frac{m}{2}\right\}$, corresponding to the vacuum $\langle 1\rangle$ with $\boldsymbol{\Sigma}=\frac{m}{\sqrt{3}}(1,-1,-1)$. Similarly, the green, cyan, and yellow regions correspond to the vacua $\langle 2\rangle,\langle 3\rangle$, and $\langle 4\rangle$, respectively. Note that we only show the interior of the sphere of the radius 10 with the unit of $m^{-1}$. Figure 6(b) shows a superposition of six domain walls dividing the four vacua, where the orange part is the region inside which $\sum_{m=1}^{3} \mathcal{Z}_{m}>\frac{1}{2 v^{2} m^{2}}$ holds. Figure 6(c) shows a superposition of four domain wall junctions of the six domain walls, where the gray part is the region inside which $\sum_{m>n} \mathcal{Y}_{m n}<-\frac{9}{100} \frac{m^{4}}{e^{2}}$ holds.

Let us next describe the exact solution on the internal $\boldsymbol{\Sigma}$ plane. As already shown in Fig. 5(a), the vacua correspond to the four vertices of the regular tetrahedron. Then, from our experience with the $\mathcal{S}_{3} / \mathcal{S}_{2}$ domain wall junction, we naturally expect that the six edges and four faces correspond to the six domain walls and the four domain wall 

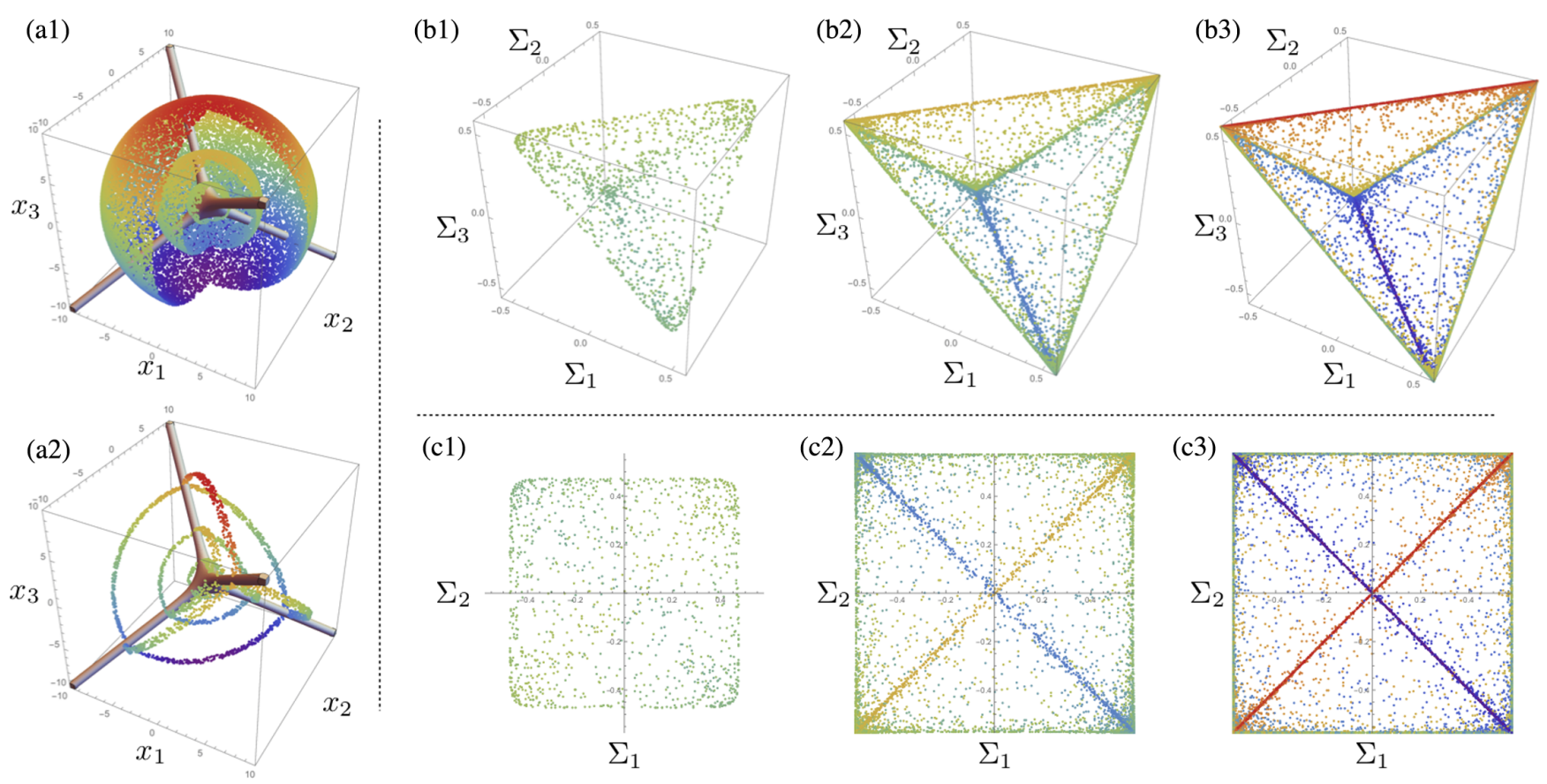

FIG. 7. (a1) The 1200, 7500, and 30,000 sampling points on the spheres of radii $r=2,5$, 10. (a2) The sampling points only inside the domain walls are shown. (b1)-(b3) The images of the sampling points of (a1). (c1)-(c3) The orthographic projections of (b1)-(b3) onto the $\Sigma_{1}-\Sigma_{2}$ plane.

junctions, respectively. To confirm this, we show the map from the internal $\boldsymbol{\Sigma}$ space to the real $\boldsymbol{x}$ space by $\boldsymbol{\Sigma}(\boldsymbol{x})$ in Fig. 7 which is the 3D extension of Fig. 4. We take sampling points randomly on the three spheres with radius $r=2,5,10$ in the real $x$ space as shown in Fig. 7(a1). The number of the sampling points are 1200,7500, and 30,000, respectively. Their images on the internal $\boldsymbol{\Sigma}$ space are shown in Figs. 7(b1)-7(b3). We can see that generic points on the spheres are mapped onto either of the vertices of the tetrahedron. The population on the edges are much less than those on the vertices since inverse images of points on the edges are arcs [Fig. 7(a2)] which are intersections of the sampling spheres and the domain walls. The sampling points inside the domain wall junctions are mapped onto the faces of the tetrahedron. Clearly, there are much rare points on the faces. Comparing (b1)-(b3), one sees that the tetrahedral shape becomes clearer for the lager sphere. We also show orthographic projections of the tetrahedral images onto the $\Sigma_{1}-\Sigma_{2}$ plane as Figs. 7(c1)-7(c3).

As we have learned above, there is a good relation between the configuration expressed in the $\boldsymbol{x}$ space and that in the $\boldsymbol{\Sigma}$ space. As is summarized in Table I, the vacua, domain walls, and the domain wall junctions correspond to the 3D domains, the 2D planes, and the 1D lines in the $\boldsymbol{x}$ space, and to the vertices, the edges, and the faces of the tetrahedron in the $\boldsymbol{\Sigma}$ space. At this point, we realize that there is one more piece, namely, the junction of the domain wall junctions in the $\boldsymbol{x}$ space, which is a pointlike object. Its natural counterpart in the $\boldsymbol{\Sigma}$ space should be 3D interior of the tetrahedron. This can be already seen in Fig. 7(b1) where the small sphere near the junction of the domain wall junctions is mapped on the tetrahedral inner surface. In order to make a more rigorous argument, we return to the Bogomol'nyi completion in Eq. (3.3). There, we found the two topological quantities $\mathcal{Z}_{m}$ and $\mathcal{Y}_{m n}$ for the domain walls and the domain wall junctions, respectively. No other topological objects take part. However, we now realize that $\mathcal{Z}_{m}$ is the $1 \mathrm{D}$ Jacobian between $x_{m}$ and $\Sigma_{m}$, and $\mathcal{Y}_{m n}$ is the 2D Jacobian between $\left\{x_{m}, x_{n}\right\}$ and $\left\{\Sigma_{m}, \Sigma_{n}\right\}$. Along this line, we naturally define a new topological quantity as the three-dimensional Jacobian between $\left\{x_{1}, x_{2}, x_{3}\right\}$ and $\left\{\Sigma_{1}, \Sigma_{2}, \Sigma_{3}\right\}$ by

TABLE I. Duality among the constituents of the $\mathcal{S}_{4} / \mathcal{S}_{3}$ three-dimensional domain wall junction in the $\boldsymbol{x}$ space and the $\boldsymbol{\Sigma}$ space.

\begin{tabular}{lcccc}
\hline \hline & Vac & DW & DW junction & Junction of DW junctions \\
\hline $\boldsymbol{x}$ space & 3D domain & Plane (2D) & Line (1D) & Point (0D) \\
$\boldsymbol{\Sigma}$ space & Vertex (0D) & Edge (1D) & Face (2D) & Interior (3D) \\
Degree & 4 & 6 & 4 & 1 \\
\hline \hline
\end{tabular}




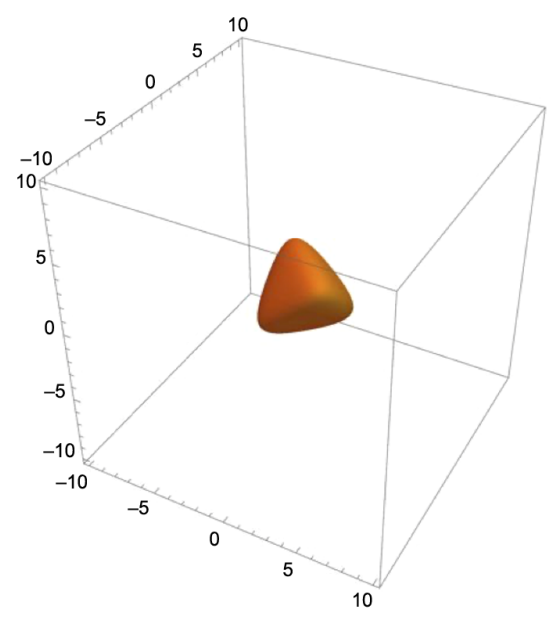

(a) $\mathcal{X}_{123}$ Junction of DW junctions

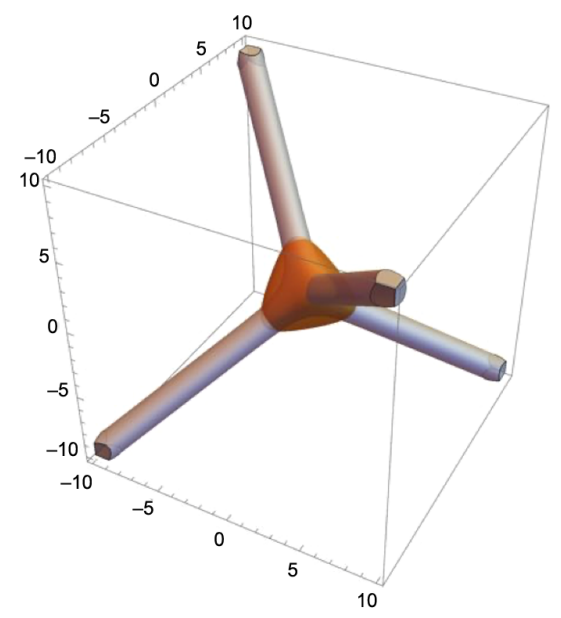

(b) $\mathcal{X}_{123}$ and $\mathcal{Y}_{12}+\mathcal{Y}_{23}+\mathcal{Y}_{31}$

FIG. 8. The topological charge density $\mathcal{X}_{123}$ of the junction of the domain wall junctions. (a) shows the constant-level surface with $\mathcal{X}_{123}$ and (b) shows superposition of Figs. 8(a) and 6(c).

$$
\mathcal{X}_{123} \equiv \operatorname{det}\left(\begin{array}{lll}
\partial_{1} \Sigma_{1} & \partial_{1} \Sigma_{2} & \partial_{1} \Sigma_{3} \\
\partial_{2} \Sigma_{1} & \partial_{2} \Sigma_{2} & \partial_{2} \Sigma_{3} \\
\partial_{3} \Sigma_{1} & \partial_{3} \Sigma_{2} & \partial_{3} \Sigma_{3}
\end{array}\right)
$$

Note that this can be cast into the total derivative form as

$$
\mathcal{X}_{123}=\partial_{l}\left(\epsilon^{l m n} \Sigma_{1} \partial_{m} \Sigma_{2} \partial_{n} \Sigma_{3}\right),
$$

with $m, n, l$ run from 1 to 3 . Therefore, this is a topological charge density. For the $\mathcal{S}_{4}$ symmetric solution, we have

$$
X=\int d^{3} x \mathcal{X}_{123}=\frac{1}{3}\left(\frac{2 m}{\sqrt{3}}\right)^{3}
$$

This corresponds to the volume of the tetrahedron in the $\boldsymbol{\Sigma}$ space. Thus, the $X$ divided by the reference tetrahedron volume represents the topological charge 1 of the map.

Figure 8 shows a 3D constant-level surface with $\mathcal{X}_{123}=$ $\frac{1}{100}$, which is indeed a tetrahedral blowup of the junction point of the domain wall junctions in the $\boldsymbol{x}$ space.

\section{EXACT SOLUTIONS OF $\mathcal{S}_{D+1} / \mathcal{S}_{D}$ DOMAIN WALL JUNCTIONS}

\section{A. Deriving exact solutions in generic dimensions}

The derivation of the exact solutions of the domain wall junctions in Secs. IV and V can be generalized straightforwardly to higher dimensions. To this end, let us consider the $(D+1)$-dimensional model with $N_{F}^{\prime}=D$ and $N_{F}=$ $D+1$ (we have studied $D=2,3$ in Secs. IV and V).

We will prove that the $\frac{1}{2^{D}}$ BPS equations (3.13)-(3.16) admit an exact solution

$$
A_{\mu}=0, \quad \Sigma_{m}=\partial_{m} \phi, \quad H_{A}=v e^{-\phi_{w_{A}},}
$$

where $m=1,2, \ldots, D$ and $A=1,2, \ldots, D+1$, and $w_{A}$ and $\phi$ are given by

$$
w_{A}=e^{\boldsymbol{m}_{A} \cdot \boldsymbol{x}}, \quad \phi=\log \sum_{A=1}^{D+1} w_{A},
$$

and the mass vectors $\boldsymbol{m}_{A}$ should correspond to the coordinates of $D+1$ vertices of a regular $D$-simplex of the radius $m$, namely, $\left|\boldsymbol{m}_{A}\right|=m$. One can easily show that Eqs. (3.13), (3.14), and (3.16) are solved by Eqs. (6.1) and (6.2). Then, it will turn out that a highlight of the proof is verifying Eq. (3.15) which is written as

$$
\frac{1}{e^{2} v^{2}} \triangle_{D}^{2} \phi=1-e^{-2 \phi} \psi, \quad \psi \equiv \sum_{A=1}^{D+1} w_{A}^{2},
$$

only if the relation

$$
e v=\sqrt{\frac{D+1}{D}} m
$$

holds. This is the first solution for the domain wall junction in generic $D$ dimensions.

One of the less beautiful parts in the following argument comes from complexity of giving the coordinate of each vertex of the regular $D$-simplex in the internal $D$-dimensional space; see, for example, Eq. (2.10) for $D=2$ and Eqs. (5.4)(5.7) for $D=3$. In order to avoid this inessential complexity, we here embed the problem of the $D$-dimensional real and internal spaces into the $D+1$-dimensional ones. Namely, we consider $\mathcal{L}$ with the spacetime dimensions $(D+1)+1$ and the flavor number $N_{F}^{\prime}=D+1$, with keeping $N_{F}=D+1$. Namely, we leave the $D$-simplex as it is (we do not consider a $D+1$-simplex); see Fig. 9 for the case of 2-simplex before and after the embedding. There are 


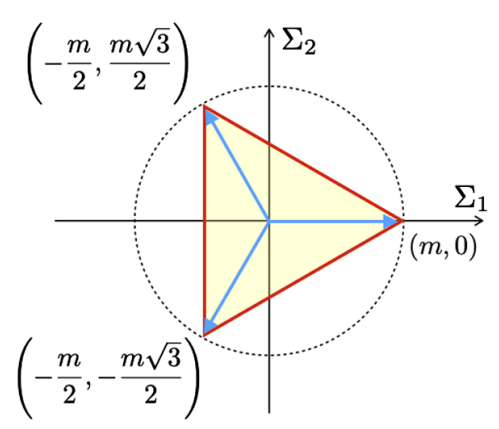

2-simplex in $D=2$

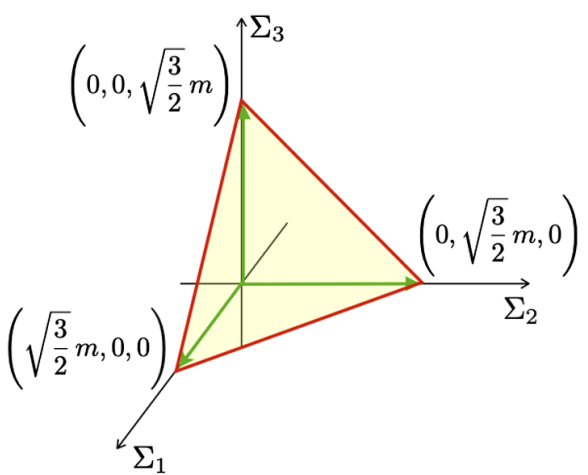

2-simplex in $D=3$

FIG. 9. A regular 2-simplex (an equilateral triangle) drawn in $D=2$ and $D=3$ spaces.

two advantages to do this. One is that the mass vectors (corresponding to the vertices of the regular $D$-simplex) can be simply expressed as

$$
\begin{gathered}
\boldsymbol{m}_{1}=m^{\prime}(1,0,0, \ldots, 0,0), \\
\boldsymbol{m}_{2}=m^{\prime}(0,1,0, \ldots, 0,0), \\
\vdots \\
\boldsymbol{m}_{D}=m^{\prime}(0,0,0, \ldots, 1,0), \\
\boldsymbol{m}_{D+1}=m^{\prime}(0,0,0, \ldots, 0,1),
\end{gathered}
$$

with

$$
m^{\prime}=\sqrt{\frac{D+1}{D}} m
$$

The second merit is that the discrete symmetry among these vectors is clearly the $\mathcal{S}_{D+1}$ group which is identical to permutations of the $D+1$ axes. Thanks to the first advantage, $w_{A}$ becomes drastically simple as

$$
w_{A}=e^{m^{\prime} x^{A}}, \quad(A=1,2, \ldots, D+1) .
$$

Then, we can easily verify that $\phi$ given in Eq. (6.2) satisfies the following equation:

$$
\triangle_{D+1} \phi=m^{\prime 2}\left(1-e^{-2 \phi} \psi\right) .
$$

This is identical to Eq. (6.3) with Eq. (6.4) only except for the Laplacian $\triangle_{D+1}$ instead of $\triangle_{D}$. To complete the proof, we need to show the equivalence between Eqs. (6.3) and (6.11). This can be done as follows. First, we note that any $D$ simplex is included in a $D$-dimensional subspace of the $D+1$-dimensional space. However, we used the $D+1$ vectors $\boldsymbol{m}_{A}(A=1,2, \ldots, D+1)$ for expressing the $D+1$ vertices of the regular $D$-simplex as in Eqs. (6.5)-(6.8).
Therefore, there exists an appropriate $U \in S O(D+1)$ transformation which transforms the vectors as

$$
\boldsymbol{m}_{A} \rightarrow \tilde{\boldsymbol{m}}_{A}=U \boldsymbol{m}_{A}=m^{\prime}\left(\tilde{m}_{1, A}, \ldots, \tilde{m}_{D, A}, 0\right), \quad \text { for all } A
$$

At the same time, we can also redefine the extended $D+1$ dimensional spacial coordinate by the same $S O(D+1)$ element by

$$
x \rightarrow \tilde{x}=U x .
$$

This transformation does not change the inner product

$$
\boldsymbol{m}_{A} \cdot \boldsymbol{x}=\tilde{\boldsymbol{m}}_{A}^{T} U^{T} U \tilde{\boldsymbol{x}}=\tilde{\boldsymbol{m}}_{A} \cdot \tilde{\boldsymbol{x}} .
$$

Since the $(D+1)$ th component of $\tilde{\boldsymbol{m}}_{A}(A=1,2, \ldots, D+1)$ is zero, the $(D+1)$ th coordinate $\tilde{x}_{D+1}$ does not appear in Eq. (6.14). Both $\phi$ and $\psi$ include $\tilde{\boldsymbol{x}}$ only through the combination $\boldsymbol{m}_{A} \cdot \boldsymbol{x}=\tilde{\boldsymbol{m}}_{A} \cdot \tilde{\boldsymbol{x}}$, so that $\tilde{x}_{D+1}$ is completely redundant. Hence, the $(D+1)$-dimensional Laplacian in terms of the new coordinate $\tilde{\boldsymbol{x}}$ is identical to the $D$-dimensional one in Eq. (6.11).

We again have faced the power of the scalar potential $\phi$. All the procedures to obtain the exact analytic solutions for generic $D$ are straightforward extension of those developed in this work for $D=2$ in the previous section, together with the correct understanding of the discrete symmetry $\mathcal{S}_{D+1}$. Without these developments, we could not find the analytic solutions.

\section{B. Geometric properties}

We now have the picture that the $\mathcal{S}_{D+1} / \mathcal{S}_{D}$ domain wall junction in $D$-dimensional real space is dual to the regular $D$-simplex. Due to this correspondence, we can easily understand the physical structure of the higher dimensional domain wall junctions. In mathematics, the convex hull of a subset of size $d+1$ of the $D+1$ points of the $D$-simplex is 
TABLE II. The number of $d$-faces ( $d$-walls) of $D$-simplex for $D=1,2,3,4,5,6$.

0 -face 1 -face 2 -face 3 -face 4 -face 5 -face 6 -face

1-simplex $2 \quad 1$

2-simplex $3 \quad 3$

3-simplex 46

4-simplex $5 \quad 10$

5-simplex $6 \quad 15$

6-simplex 7

$21 \quad 35$

1

$4 \quad 1$

$10 \quad 5$

$20 \quad 15$

0-wall 1-wall 2-wall 3-wall 4-wall 5-wall 6-wall

called a $d$-face of the $D$-simplex (the 0 -faces are the vertices, the 1 -faces are the edges, and the $(D-1)$-faces are called the facets). The number of the $d$-faces is the binomial coefficient $\left(\begin{array}{c}D+1 \\ d+1\end{array}\right)$. Several low-lying examples are shown in Table II. In the physics context, by the duality, we relate the 0 -faces to the $D$-dimensional vacuum domains, the 1-faces to the $(D-1)$-dimensional domain walls, and the 2-faces to the $(D-2)$-dimensional domain wall junctions, and so on. We call the $d$-faces the $d$-walls. Namely, the 0 -wall means the vacuum, the 1 wall corresponds to the domain wall, and the 2-wall stands for the domain wall junction, and so on. We have already studied the 2- and 3-simplexes in Secs. IV and V, respectively.

Let us introduce a topological quantity to each of the $d$-wall for $d \geq 1$. For $d=1$, the natural quantity is $\mathcal{Z}_{m}$ defined in Eq. (3.4) and its integration over the $x_{m}$ coordinate. Similarly, we ran into $\mathcal{Y}_{m n}$ in Eq. (3.5) and its integral over the $x_{m}-x_{n}$ plane for the 2-wall for $d=2$. Note that the numbers of $\mathcal{Z}_{m}$ and $\mathcal{Y}_{m n}$ of the $\mathcal{S}_{D+1} / \mathcal{S}_{D}$ domain wall junction are $D$ and $\frac{(D-1) D}{2 !}$, respectively, which coincide with $\left(\begin{array}{l}D \\ d\end{array}\right)$ for $d=1$ and $d=2$, respectively. For $d=3$, we have encountered $\mathcal{X}_{123}$ given in Eq. (5.16) for the $\mathcal{S}_{4} / \mathcal{S}_{3}$ domain wall junction in the model with $D=3$. Having $\mathcal{Z}_{m}$ and $\mathcal{Y}_{m n}$ for generic $\mathcal{S}_{D+1} / \mathcal{S}_{D}$ domain wall junction together with $\mathcal{X}_{123}$ of the $\mathcal{S}_{4} / \mathcal{S}_{3}$ domain wall junction at hand, we are now ready to define the topological quantity of the level $d$ by

$$
\begin{aligned}
& \mathcal{W}_{d}\left(m_{1}, m_{2}, \ldots, m_{d}\right) \\
& =\operatorname{det}\left(\begin{array}{cccc}
\partial_{m_{1}} \Sigma_{m_{1}} & \partial_{m_{1}} \Sigma_{m_{2}} & \cdots & \partial_{m_{1}} \Sigma_{m_{d}} \\
\partial_{m_{2}} \Sigma_{m_{1}} & \partial_{m_{2}} \Sigma_{m_{2}} & \cdots & \partial_{m_{2}} \Sigma_{m_{d}} \\
\vdots & & \ddots & \vdots \\
\partial_{m_{d}} \Sigma_{m_{1}} & \partial_{m_{d}} \Sigma_{m_{2}} & \cdots & \partial_{m_{d}} \Sigma_{m_{d}}
\end{array}\right)
\end{aligned}
$$

where $m_{\alpha} \in\{1,2, \ldots, D\}(\alpha=1,2, \ldots, d)$ and $m_{\alpha}>m_{\beta}$ if $\alpha>\beta$. The number of the level- $d \mathcal{W}$ is $\left(\begin{array}{l}D \\ d\end{array}\right)$; see Table III for several low-lying $D=1,2,3,4,5,6$. Note that this can be cast into the total derivative form. For example, $\mathcal{W}_{D}$ can be expressed as

$$
\mathcal{W}_{D}=\partial_{m_{1}}\left(\epsilon^{m_{1} \cdots m_{D}} \Sigma_{1} \partial_{m_{2}} \Sigma_{2} \partial_{m_{3}} \Sigma_{3} \cdots \partial_{m_{D}} \Sigma_{D}\right)
$$

Thus, this is a topological charge density. The same can be said for $\mathcal{W}_{d}$ for all $d \leq D$.

By definition, the level- $d$ wall charge coincides with the volume of a $d$-face of the $D$-simplex inscribed by a $D$-sphere of radius $m$ [the side length is $\sqrt{2(D+1) / D} m$ defined in Eqs. (6.5)-(6.8)],

$$
\begin{aligned}
& W_{d}\left(m_{1}, m_{2}, \ldots, m_{d}\right) \\
& \quad=\int d x_{m_{1}} d x_{m_{2}} \cdots d x_{m_{d}}\left|\mathcal{W}\left(m_{1}, m_{2}, \ldots, m_{d}\right)\right| \\
& \quad=\left(\sqrt{\frac{2(D+1)}{D}} m\right)^{d} \frac{\sqrt{d+1}}{d ! 2^{\frac{d}{2}}} .
\end{aligned}
$$

The level-1 $\mathcal{W}_{1}\left(x_{m}\right)$ and the level-2 $\mathcal{W}_{2}\left(x_{m}, x_{n}\right)$ are related to $\mathcal{Z}_{m}$ and $\mathcal{Y}_{m n}$, respectively. Therefore, as in the lower dimensional case, with appropriately normalized, $W_{d}$ represents the degree of the map counting how many times the $d$-dimensional subpolytope is covered when we sweep the $d$-dimensional subspace $x_{m_{1}}-x_{m_{1}}-\cdots-x_{m_{d}}$ once.

Before closing this section, let us briefly sketch a higher dimensional junction which we have not shown yet. The first example is the $\mathcal{S}_{5} / \mathcal{S}_{4}$ domain wall junction (the 4-simplex) in the model with $N_{F}=5$ and $D=N_{F}^{\prime}=4$. A concrete set of the five mass vectors is given by

TABLE III. The number of level- $d \mathcal{W}$ of $\mathcal{S}_{D+1} / \mathcal{S}_{D}$ domain wall junction for $D=1,2,3,4,5,6$.

\begin{tabular}{ccccccc}
\hline \hline & Level-1 $\mathcal{W}$ & Level-2 $\mathcal{W}$ & Level-3 $\mathcal{W}$ & Level-4 $\mathcal{W}$ & Level-5 $\mathcal{W}$ & Level-6 $\mathcal{W}$ \\
\hline $\mathcal{S}_{2}$ DW & 1 & & & & & \\
$\mathcal{S}_{3} / \mathcal{S}_{2}$ DWJ & 2 & 1 & & & & \\
$\mathcal{S}_{4} / \mathcal{S}_{3}$ DWJ & 3 & 3 & 1 & & & \\
$\mathcal{S}_{5} / \mathcal{S}_{4}$ DWJ & 4 & 6 & 4 & 1 & & \\
$\mathcal{S}_{6} / \mathcal{S}_{5}$ DWJ & 5 & 10 & 10 & 5 & 1 & 1 \\
$\mathcal{S}_{7} / \mathcal{S}_{6}$ DWJ & 6 & 15 & 20 & 15 & 6 & \\
& $\mathcal{Z}_{m}$ & $\mathcal{Y}_{m n}$ & $\mathcal{X}_{l m n}$ & & & \\
\hline \hline
\end{tabular}



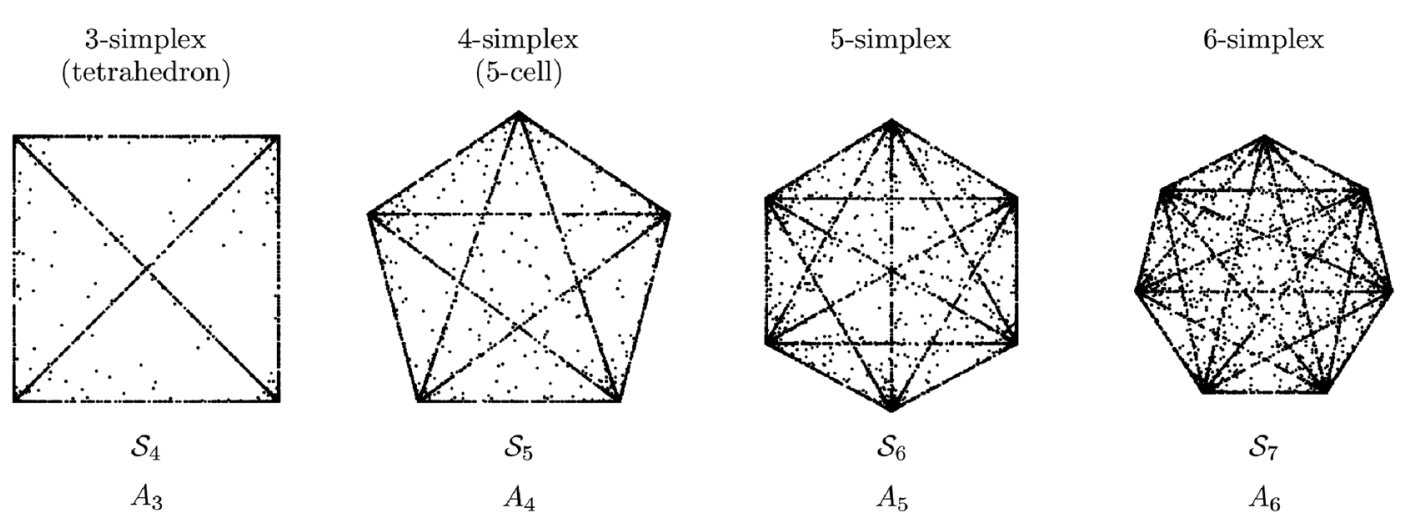

FIG. 10. Orthographic projections of images for randomly chosen 20,000 points on a $(D-1)$-sphere (radius $|\boldsymbol{x}|=40)$ by the exact solutions of $\mathcal{S}_{D+1} / \mathcal{S}_{D}$ domain wall junctions with $D=3,4,5,6$. The diagrams are identical to the so-called Coxeter plane of type $A_{D}$ for a regular $D$-simplex.

$$
\begin{gathered}
\boldsymbol{m}_{1}=\frac{\sqrt{5}}{4} m\left(1,-1,-1,-\frac{1}{\sqrt{5}}\right), \\
\boldsymbol{m}_{2}=\frac{\sqrt{5}}{4} m\left(-1,1,-1,-\frac{1}{\sqrt{5}}\right), \\
\boldsymbol{m}_{3}=\frac{\sqrt{5}}{4} m\left(-1,-1,1,-\frac{1}{\sqrt{5}}\right), \\
\boldsymbol{m}_{4}=\frac{\sqrt{5}}{4} m\left(1,1,1,-\frac{1}{\sqrt{5}}\right) \\
\boldsymbol{m}_{5}=\frac{\sqrt{5}}{4} m\left(0,0,0, \frac{4}{\sqrt{5}}\right) .
\end{gathered}
$$

The corresponding $\mathcal{S}_{5} / \mathcal{S}_{4}$ domain wall junction divides $D=4$ space into five vacuum domains. It is not easy to imagine such noncompact higher dimensional object, but, instead, all the geometric data can be easy read from Table II. As a supplementary, we show the images on a twodimensional plane in $\boldsymbol{\Sigma}$ space whose preimages are randomly chosen 20,000 points on the 3 -sphere $S^{3}$ of the radius $|\boldsymbol{x}|=40$ in Fig. 10. Almost all points are mapped on either of five vertices ( 0 -walls) of the pentagon, and the much less points are mapped on the ten edges (1-wall) of the pentagon and pentagram. Much rarer points are mapped onto interior of the pentagon. These two-dimensional images, the pentagon and pentagram, are familiar for the 5-cell. It is a orthographic projection of the 5-cell onto a two-dimensional plane (the so-called Coxeter plane). The pentagon with pentagram is the type $A_{4}$ Coxeter plane in which all the vertices and edges of 5-cells are separately shown. The symbol $A_{4}$ comes from the fact that the symmetric group $\mathcal{S}_{5}$ of the 5-cell is isomorphic to the Coxeter group of the type $A_{4}$.

The symmetry group $\mathcal{S}_{D+1}$ of a regular $D$-simplex is known as the Coxeter group of type $A_{D}$, and its Coxeter plane is known as a convex regular $(D+1)$-gon. Our exact solution of the $\mathcal{S}_{D+1} / \mathcal{S}_{D}$ domain wall junction gives the Coxeter plane of type $A_{D}$ via the map from the $D$-dimensional real space $\boldsymbol{x}$ to the $D$-dimensional internal space $\boldsymbol{\Sigma}$. We show several concrete diagrams for $D=3,4$, 5, 6 in Fig. 10.

\section{SUMMARY AND DISCUSSION}

In this work, we have constructed the exact solutions of the $\mathcal{S}_{D+1} / \mathcal{S}_{D}$ domain wall junctions in $D+1$-dimensional spacetime. We have considered SUSY motivated Abelian gauge theories with $N_{F}$ charged complex scalar fields $H_{A}$ and $N_{F}^{\prime}=D$ real scalar fields $\Sigma_{A^{\prime}}$ and have derived the new BPS equations for the domain wall junctions. We then have restricted ourselves to the cases with specific flavor numbers $N_{F}=D+1$ and have obtained the analytic exact BPS solutions in the extended Abelian-Higgs model for the first time. There are two necessary conditions for finding the exact solutions. The first is the $\mathcal{S}_{D+1}$ symmetric masses $\boldsymbol{m}_{A}$ so that the mass vectors should be placed at vertices of a regular $D$-simplex in the internal space. The other is the special relation between the coupling constants given in Eq. (6.4). When these conditions are satisfied, we find that the exact BPS solutions can be obtained by the scalar potential $\phi$ as in Eqs. (6.1) and (6.2). We should emphasize that introducing the scalar potential $\phi$ has been crucial to reformulate the complicated BPS equations to be surprisingly simple. We have been able to accomplish constructing the analytic solutions with the aid of $\phi$. We have verified that the solution for $D=2$ is identical to the one previously obtained in Ref. [80] in which the scalar potential $\phi$ was not used. We also have developed how to describe such noncompact extended solitons in higher dimensional spaces. We have found that there is a one-toone correspondence between the BPS configuration in the real $\boldsymbol{x}$ space and that in the internal $\boldsymbol{\Sigma}$ space. The latter is more useful and tractable because it deals with compact objects, the regular $D$-simplex. This correspondence allows 
us to understand what kind of intersections are included. We have found that all the building blocks are in one-to-one correspondence to the $d$-faces (we called them the $d$-walls) of the regular $D$-simplex. Thus, the $\mathcal{S}_{D+1} / \mathcal{S}_{D}$ domain wall junction consists of $\left(\begin{array}{c}D+1 \\ d+1\end{array}\right) d$-walls; see Table II. The Bogomol'nyi completion shows that the energy density of the $\mathcal{S}_{D+1}$ domain wall junctions depends on only two kinds of the topological charge densities, the domain wall (1-wall) charge $Z_{m}$ and the domain wall junction (2-wall) charge $Y_{m n}$. The topological charge densities $\mathcal{Z}_{m}$ and $\mathcal{Y}_{m n}$ are a one-dimensional Jacobian from $x_{m}$ to $\Sigma_{m}$ and a twodimensional Jacobian from $x_{m}-x_{n}$ to $\Sigma_{m}-\Sigma_{n}$, respectively. Then, we have been naturally led to extend them for the generic $d$-walls by the $d$-dimensional Jacobian from the real $\mathbb{R}^{d}$ space to the internal $\mathbb{R}^{d}$ space. The simplest example is $X(d=3)$ for the tetrahedral domain wall junction $(D=3)$; see Fig. 8. All the topological charges for the generic $d$-walls have been unified by the level- $d$ wall charge $W_{d}$ in Eq. (6.15). The final achievement in this work is on the visualization of the $\mathcal{S}_{D+1} / \mathcal{S}_{D}$ domain wall junctions. Describing the regular $D$-simplex in lower dimensions is known as the Coxeter plane. It is a twodimensional projection on a convex regular $(D+1)$-gon which is the Coxeter diagram of type $A_{D}$. We also find that the exact solutions $\boldsymbol{\Sigma}(\boldsymbol{x})$ are canonical mappings from the former to the regular $D$-simplex in the $\boldsymbol{\Sigma}$ space, and their images are nothing but the Coxeter diagram of the type $A_{D}$.

We have assumed the special relation among the parameters $e, v$, and the masses, in order to find the analytic solutions of the newly found BPS equations in generic $D$ dimensions. The models are limited, however, with the analytic solutions at our hands; now we clearly have understood the whole picture of the domain wall junctions in higher dimensions. We have been able to easily explain how many $d$-walls are included in the $D$-dimensional junction. Since these properties are topological, they do not change even when we continuously deform the model parameters. For generic parameters, there are no analytic solutions and therefore one needs to solve numerically the complicated differential equations in $D$ dimensions.

Before closing this paper, let us mention several future directions. First, we have considered only $\mathcal{S}_{D+1}$ symmetric masses corresponding to vertices of the regular $D$-simplex to construct the exact solutions. If we consider more general masses corresponding to a deformed $D$-simplex, the corresponding the domain wall junction is also deformed from the $\mathcal{S}_{D+1}$ symmetric configuration. For such generic case, we cannot expect the existence of analytic solutions for generic couplings, but in the strong coupling limit analytic solutions would be available as for the $D=2$ case [81]. For generic dimensions, we have exhausted all exact solutions with full moduli parameters in a separated paper [94]. More generally, we would need numerical works to obtain the BPS solutions for generic couplings.
Second, we have studied the minimal models in which the flavor number of the charged scalar fields is chosen as $N_{F}=D+1$. When we increase the number $N_{F}$ of flavors, the number of the vacua increases accordingly. This leads to generic polytopes other than the $D$-simplex which correspond to more complicated networks in the real $\boldsymbol{x}$ space. In particular, it admits domain wall loops for $D=2$ [81], in which case the low energy effective action was constructed for localized modes of domain wall loops [84], and the low energy dynamics of such loops was studied in the moduli approximation [85]. The same can be done for higher dimensional extensions in this paper, which would admit higher dimensional loops, like holes surrounded by domain walls.

Third, we can extend the Abelian gauge theory studied in this paper to $U\left(N_{C}\right)$ gauge theories. For the $D=2$ case, it was found that the non-Abelian extension of the junction charge $\mathcal{Y}_{12}$ exists in the non-Abelian gauge theories [81]. In such a case, if some masses are degenerated, there appear non-Abelian moduli on the domain walls, called nonAbelian clouds [47,52,54]. Such non-Abelian moduli would also appear in the domain wall junctions in general dimensions.

Fourth, we can consider non-BPS domain wall junction in $D+1$-dimensional spacetime, while in this paper, we have concentrated only on the BPS solutions. Once we relax the BPS condition, we would have more generic networks of the domain walls, as for the simplest WessZumino model [90].

Fifth, as for a possible connection with differential geometry, the case of $D=2$ was found to be interpreted in terms of tropical geometry and amoeba in mathematics [86]. Higher dimensional extensions of the present paper will provide higher dimensional correspondence to tropical geometry and amoeba.

Finally, let us mention possible applications of our model to physics. Single or parallel domain walls can be applied to Josephson junction arrays of superconductors sandwiching insulators $[95,96]$. This can be extended to three-dimensional junction found in this paper. Also, cosmological domain wall networks [17] will be one of the most interesting physical applications.

\section{ACKNOWLEDGMENTS}

This work was supported by the Ministry of Education, Culture, Sports, Science (MEXT)-Supported Program for the Strategic Research Foundation at Private Universities "Topological Science" (Grant No. S1511006) and JSPS KAKENHI Grant No. 16H03984. The work of M. E. was also supported in part by JSPS Grant-in-Aid for Scientific Research KAKENHI Grant No. JP19K03839 and by MEXT KAKENHI Grant-in-Aid for Scientific Research on Innovative Areas "Discrete Geometric Analysis for Materials Design" Grant No. JP17H06462 from the MEXT of Japan. The work of M. N. was also supported 
in part by JSPS KAKENHI Grant No. $18 \mathrm{H} 01217$ by a Grant-in-Aid for Scientific Research on Innovative Areas "Topological Materials Science" (KAKENHI Grant No. 15H05855) from MEXT of Japan.
APPENDIX: THE SYMMETRIC GROUP $\mathcal{S}_{4}$ AND THE COSET $\mathcal{S}_{4} / \mathcal{S}_{3}$

We give the $4 \times 4$ matrix representation of $\mathcal{S}_{4}$,

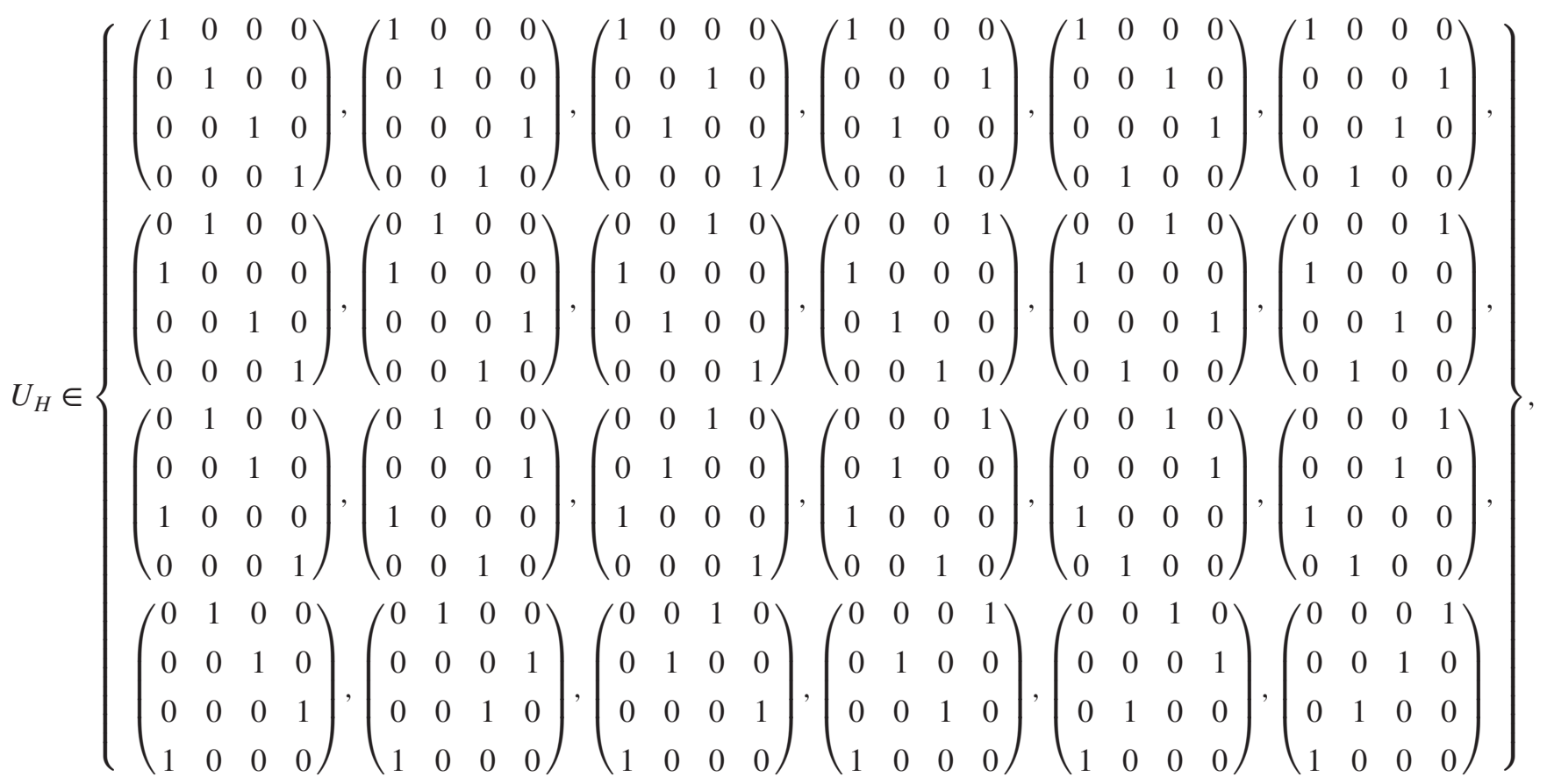

and the corresponding $3 \times 3$ representation,

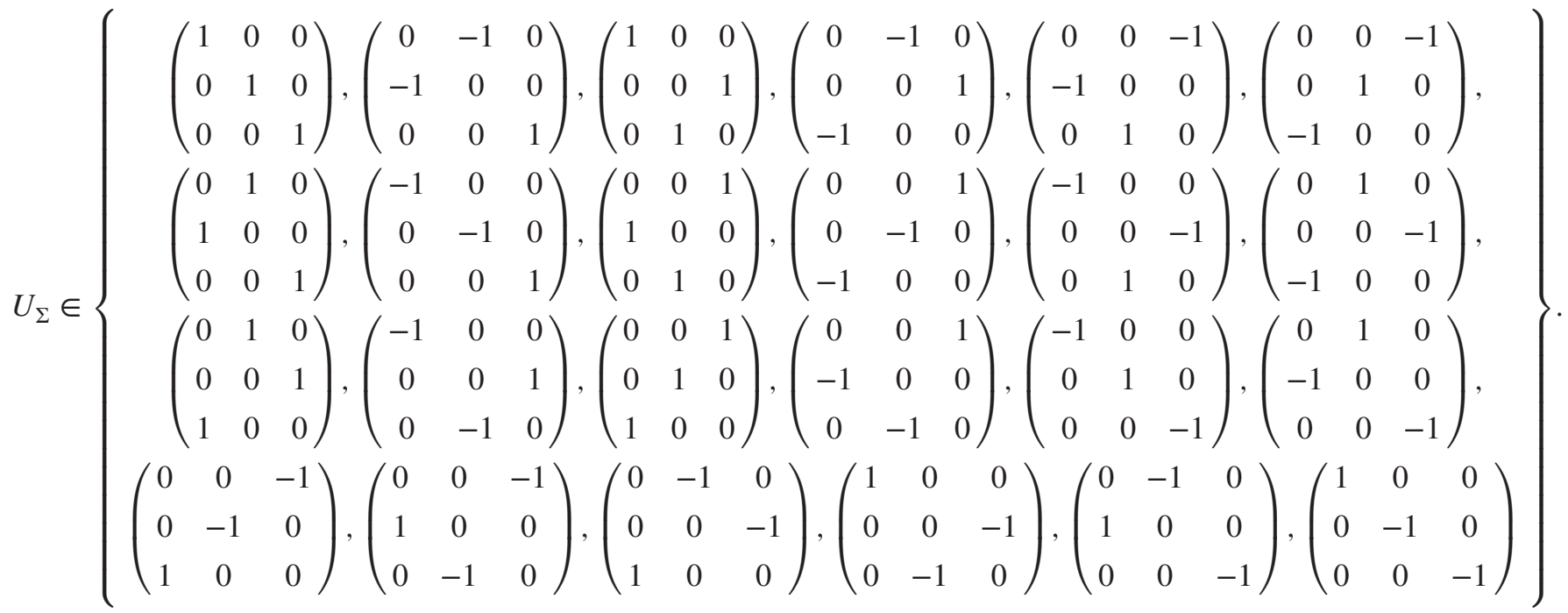

These satisfy the relation (2.8) with the mass matrix $\mathcal{M}$ given in Eq. (5.8). Note that the one-to-one correspondence between $U_{H}$ and $U_{\Sigma}$ is given by $U_{\Sigma}=\left(\mathcal{M M}^{T}\right)^{-1} \mathcal{M} U_{H}^{-1 T} \mathcal{M}^{T}$. The $4 !=24$ matrices of Eq. (A1) coincide to the standard $4 \times 4$ representation of $\mathcal{S}_{4}$. On the contrary, the $3 \times 3$ matrices in Eq. (A2) might not be familiar, but indeed they also form another representation of $\mathcal{S}_{4}$. 
The six matrices in the top row of Eq. (A1) which is a subgroup $\mathcal{S}_{3}$ in $\mathcal{S}_{4}$ leave the first vacuum intact as

$$
\left.\boldsymbol{\Sigma}\right|_{\langle 1\rangle}=\boldsymbol{m}_{1}=\frac{m}{\sqrt{3}}\left(\begin{array}{c}
1 \\
-1 \\
-1
\end{array}\right) \rightarrow U_{\Sigma} \boldsymbol{m}_{1}=\boldsymbol{m}_{1}
$$

Thus, the coset of $\mathcal{S}_{4}$ by the $\mathcal{S}_{3}$ quotient has four representatives,

$$
\mathcal{S}_{4} / \mathcal{S}_{3}=\left\{\left(\begin{array}{lll}
1 & 0 & 0 \\
0 & 1 & 0 \\
0 & 0 & 1
\end{array}\right),\left(\begin{array}{lll}
0 & 1 & 0 \\
1 & 0 & 0 \\
0 & 0 & 1
\end{array}\right),\left(\begin{array}{lll}
0 & 1 & 0 \\
0 & 0 & 1 \\
1 & 0 & 0
\end{array}\right),\left(\begin{array}{ccc}
0 & 0 & -1 \\
0 & -1 & 0 \\
1 & 0 & 0
\end{array}\right)\right\} .
$$

Note that $\mathcal{S}_{3}$ is not a normal subgroup of $\mathcal{S}_{4}$. Therefore, the coset $\mathcal{S}_{4} / \mathcal{S}_{3}$ is not a group.

[1] N. S. Manton and P. Sutcliffe, Topological Solitons, Cambridge Monographs on Mathematical Physics (Cambridge University Press, Cambridge, United Kingdom, 2004).

[2] T. Vachaspati, Kinks and Domain Walls: An Introduction to Classical and Quantum Solitons (Cambridge University Press, Cambridge, England, 2006).

[3] R. Rajaraman, Solitons and Instantons. An Introduction to Solitons and Instantons in Quantum Field Theory (North Holland, Amsterdam, the Netherlands, 1982), p. 409, ISBN 9780444862297.

[4] T. W. B. Kibble, Some implications of a cosmological phase transition, Phys. Rep. 67, 183 (1980).

[5] T. W. B. Kibble, Topology of cosmic domains, and strings, J. Phys. A 9, 1387 (1976).

[6] F. B. Humphrey, Domain Walls and Wall Structure, edited by G. C. Hadjipanayis and G. A. Prinz, Science and Technology of Nanostructured Magnetic Materials. NATO ASI Series (Series B: Physics) Vol. 259 (Springer, Boston, MA, 1991), ISBN. .

[7] L. Yin, H. Jiang, J. Qiao et al., Direct imaging of topological edge states at a bilayer graphene domain wall, Nat. Commun. 7, 11760 (2016).

[8] I. Serban, B. Béri, A. R. Akhmerov, and C. W. J. Beenakker, Domain Wall in a Chiral p-Wave Superconductor: A Pathway for Electrical Current, Phys. Rev. Lett. 104, 147001 (2010).

[9] H. Takeuchi, K. Kasamatsu, M. Tsubota, and M. Nitta, Tachyon Condensation Due to Domain-Wall Annihilation in Bose-Einstein Condensates, Phys. Rev. Lett. 109, 245301 (2012).

[10] G.E. Volovik, The Universe in a Helium Droplet (Clarendon, Oxford, 2003).

[11] W.H. Zurek, Cosmological experiments in superfluid Helium? Nature (London) 317, 505 (1985).

[12] W. H. Zurek, Cosmological experiments in condensed matter systems, Phys. Rep. 276, 177 (1996).
[13] M. Eto, K. Hashimoto, and T. Hatsuda, Ferromagnetic neutron stars: Axial anomaly, dense neutron matter, and pionic wall, Phys. Rev. D 88, 081701 (2013).

[14] S. Yasui and M. Nitta, Domain walls in neutron ${ }^{3} P_{2}$ superfluids in neutron stars, Phys. Rev. C 101, 015207 (2020).

[15] M. Eto, Y. Hirono, M. Nitta, and S. Yasui, Vortices and other topological solitons in dense quark matter, Prog. Theor. Exp. Phys. 2014, 12D01 (2014).

[16] A. Vilenkin and E.P.S. Shellard, Cosmic Strings and Other Topological Defects (Cambridge University Press, Cambridge, United Kingdom, 2000), ISBN: .

[17] M. Bucher and D. N. Spergel, Is the dark matter a solid? Phys. Rev. D 60, 043505 (1999); R. A. Battye, M. Bucher, and D. Spergel, Domain wall dominated universes, arXiv: astro-ph/9908047; A. Friedland, H. Murayama, and M. Perelstein, Domain walls as dark energy, Phys. Rev. D 67, 043519 (2003); L. Conversi, A. Melchiorri, L. MersiniHoughton, and J. Silk, Are domain walls ruled out? Astropart. Phys. 21， 443 (2004); J. C. R. E. Oliveira, C. J. A.P. Martins, and P.P. Avelino, The cosmological evolution of domain wall networks, Phys. Rev. D 71, 083509 (2005); P. P. Avelino, J.C.R.E. Oliveira, and C. J. A.P. Martins, Understanding domain wall network evolution, Phys. Lett. B 610, 1 (2005); P. P. Avelino, C. J. A. P. Martins, and J. C. R. E. Oliveira, One-scale model for domain wall network evolution, Phys. Rev. D 72, 083506 (2005); R. A. Battye, E. Chachoua, and A. Moss, Elastic properties of anisotropic domain wall lattices, Phys. Rev. D 73, 123528 (2006); R. A. Battye and A. Moss, Anisotropic perturbations due to dark energy, Phys. Rev. D 74, 041301 (2006); P. Pina Avelino, C. J. A. P. Martins, J. Menezes, R. Menezes, and J.C.R. E. Oliveira, Frustrated expectations: Defect networks and dark energy, Phys. Rev. D 73, 123519 (2006); P. P. Avelino, C. J. A. P. Martins, J. Menezes, R. Menezes, and J.C.R.E. Oliveira, Defect junctions and domain wall dynamics, Phys. Rev. D 73, 
123520 (2006); B. Carter, Stability of winding cosmic wall lattices with X type junctions, Classical Quantum Gravity 25, 154001 (2008); R. A. Battye and A. Moss, Scaling dynamics of domain walls in the cubic anisotropy model, Phys. Rev. D 74, 023528 (2006); P. P. Avelino, C. J. A. P. Martins, J. Menezes, R. Menezes, and J. C. R. E. Oliveira, Scaling of cosmological domain wall networks with junctions, Phys. Lett. B 647, 63 (2007); P. P. Avelino, C. J. A. P. Martins, and L. Sousa, Dynamics of biased domain walls and the devaluation mechanism, Phys. Rev. D 78, 043521 (2008); P. P. Avelino, C. J. A. P. Martins, J. Menezes, R. Menezes, and J. C. R. E. Oliveira, Dynamics of domain wall networks with junctions, Phys. Rev. D 78, 103508 (2008); P.P. Avelino, J.C. R. E. Oliveira, R. Menezes, and J. Menezes, The role of domain wall junctions in Carter's pentahedral model, Phys. Lett. B 681, 282 (2009); R. A. Battye and J. A. Pearson, Charge, junctions and the scaling dynamics of domain wall networks, Phys. Rev. D 82, 125001 (2010); L. Sousa and P. P. Avelino, Evolution of domain wall networks: The Press-Ryden-Spergel algorithm, Phys. Rev. D 81, 087305 (2010); P. P. Avelino and L. Sousa, Domain wall network evolution in $(N+1)$-dimensional FRW universes, Phys. Rev. D 83, 043530 (2011); R. A. Battye, J. A. Pearson, and A. Moss, X-type and Y-type junction stability in domain wall networks, Phys. Rev. D 84, 125032 (2011); A. M. M. Leite and C. J. A. P. Martins, Scaling properties of domain wall networks, Phys. Rev. D 84, 103523 (2011); A. M. M. Leite, C. J. A. P. Martins, and E. P. S. Shellard, Accurate calibration of the velocitydependent one-scale model for domain walls, Phys. Lett. B 718, 740 (2013); J. R. C. C. C. Correia, I. S. C. R. Leite, and C. J. A. P. Martins, Effects of biases in domain wall network evolution, Phys. Rev. D 90, 023521 (2014); M. F. Oliveira and C. J. A.P. Martins, Scaling properties of multitension domain wall networks, Phys. Rev. D 91, 043527 (2015); A. Lazanu, C. J. A. P. Martins, and E. P. S. Shellard, Contribution of domain wall networks to the CMB power spectrum, Phys. Lett. B 747, 426 (2015); L. Sousa and P. P. Avelino, Cosmic microwave background anisotropies generated by domain wall networks, Phys. Rev. D 92, 083520 (2015); C. J. A. P. Martins, I. Y. Rybak, A. Avgoustidis, and E. P. S. Shellard, Extending the velocity-dependent one-scale model for domain walls, Phys. Rev. D 93, 043534 (2016); J. R. C. C. C. Correia and C. J. A. P. Martins, General purpose graphics-processing-unit implementation of cosmological domain wall network evolution, Phys. Rev. E 96, 043310 (2017); J. R. C. C. C. Correia, I. S. C. R. Leite, and C. J. A. P. Martins, Effects of biases in domain wall network evolution. II. Quantitative analysis, Phys. Rev. D 97, 083521 (2018); P. P. Avelino, Parameter-free velocitydependent one-scale model for domain walls, Phys. Rev. D 101, 023514 (2020).

[18] M. Shifman and A. Yung, Supersymmetric Solitons (Cambridge University Press, Cambridge, United Kingdom, 2009).

[19] E. B. Bogomolny, Stability of classical solutions, Yad. Fiz. 24, 861 (1976) [Sov. J. Nucl. Phys. 24, 449 (1976)].

[20] M. K. Prasad and C. M. Sommerfield, An Exact Classical Solution for the 't Hooft Monopole, and the Julia-Zee Dyon, Phys. Rev. Lett. 35, 760 (1975).
[21] G. R. Dvali and M. A. Shifman, Domain walls in strongly coupled theories, Phys. Lett. B 396, 64 (1997); Erratum, Phys. Lett. B 407, 452 (1997).

[22] G. R. Dvali and M. A. Shifman, Dynamical compactification as a mechanism of spontaneous supersymmetry breaking, Nucl. Phys. B504, 127 (1997).

[23] A. Kovner, M. A. Shifman, and A. V. Smilga, Domain walls in supersymmetric Yang-Mills theories, Phys. Rev. D 56, 7978 (1997).

[24] A. V. Smilga and A. Veselov, Complex BPS Domain Walls and Phase Transition in Mass in Supersymmetric QCD, Phys. Rev. Lett. 79, 4529 (1997).

[25] B. Chibisov and M. A. Shifman, BPS saturated walls in supersymmetric theories, Phys. Rev. D 56, 7990 (1997); Erratum, Phys. Rev. D 58, 109901 (1998).

[26] V. S. Kaplunovsky, J. Sonnenschein, and S. Yankielowicz, Domain walls in supersymmetric Yang-Mills theories, Nucl. Phys. B552, 209 (1999).

[27] B. de Carlos and J. M. Moreno, Domain Walls in Supersymmetric QCD: From Weak to Strong Coupling, Phys. Rev. Lett. 83, 2120 (1999).

[28] G. R. Dvali, G. Gabadadze, and Z. Kakushadze, BPS domain walls in large $N$ supersymmetric QCD, Nucl. Phys. B562, 158 (1999).

[29] J. D. Edelstein, M. L. Trobo, F. A. Brito, and D. Bazeia, Kinks inside supersymmetric domain ribbons, Phys. Rev. D 57, 7561 (1998).

[30] M. Naganuma and M. Nitta, BPS domain walls in models with flat directions, Prog. Theor. Phys. 105, 501 (2001).

[31] M. Arai, S. Lee, and S. Shin, Walls in supersymmetric massive nonlinear sigma model on complex quadric surface, Phys. Rev. D 80, 125012 (2009).

[32] M. Arai and S. Shin, Walls of massive Káhler sigma models on $\mathrm{SO}(2 \mathrm{~N}) / \mathrm{U}(\mathrm{N})$ and $\mathrm{Sp}(\mathrm{N}) / \mathrm{U}(\mathrm{N})$, Phys. Rev. D 83, 125003 (2011).

[33] M. Nitta and S. Sasaki, BPS states in supersymmetric chiral models with higher derivative terms, Phys. Rev. D 90, 105001 (2014).

[34] S. B. Gudnason, M. Nitta, and S. Sasaki, BPS pion domain walls in the supersymmetric chiral Lagrangian, Phys. Rev. D 94, 025003 (2016).

[35] B. H. Lee, C. Park, and S. Shin, Vacua and walls of massdeformed Kähler nonlinear sigma models on $S O(2 N) /$ $U(N)$, Phys. Rev. D 96, 105017 (2017).

[36] M. Arai, A. Golubtsova, C. Park, and S. Shin, Vacua and walls of mass-deformed Kähler nonlinear sigma models on $S p(N) / U(N)$, Phys. Rev. D 97, 105012 (2018).

[37] E. R. C. Abraham and P. K. Townsend, $Q$ kinks, Phys. Lett. B 291, 85 (1992).

[38] E. R. C. Abraham and P. K. Townsend, More on $Q$ kinks: A $(1+1)$-dimensional analog of dyons, Phys. Lett. B 295, 225 (1992).

[39] J. P. Gauntlett, D. Tong, and P. K. Townsend, Supersymmetric intersecting domain walls in massive hyperKahler sigma models, Phys. Rev. D 63, 085001 (2001).

[40] J. P. Gauntlett, D. Tong, and P. K. Townsend, Multidomain walls in massive supersymmetric sigma models, Phys. Rev. D 64, 025010 (2001).

[41] D. Tong, The Moduli space of BPS domain walls, Phys. Rev. D 66, 025013 (2002). 
[42] K. S. M. Lee, An index theorem for domain walls in supersymmetric gauge theories, Phys. Rev. D 67, 045009 (2003).

[43] M. Arai, M. Naganuma, M. Nitta, and N. Sakai, Manifest supersymmetry for BPS walls in $N=2$ nonlinear sigma models, Nucl. Phys. B652, 35 (2003).

[44] M. Arai, M. Naganuma, M. Nitta, and N. Sakai, BPS wall in $N=2$ SUSY nonlinear sigma model with Eguchi-Hanson manifold, in A Garden of Quanta, edited by A. Arai et al. (World Scientific Publishing Co. Pte. Ltd., Singapore, 2003), pp. 299-325.

[45] A. Losev and M. Shifman, $N=2$ sigma model with twisted mass and superpotential: Central charges and solitons, Phys. Rev. D 68, 045006 (2003).

[46] Y. Isozumi, K. Ohashi, and N. Sakai, Exact wall solutions in five-dimensional SUSY QED at finite coupling, J. High Energy Phys. 11 (2003) 060.

[47] M. Shifman and A. Yung, Localization of non-Abelian gauge fields on domain walls at weak coupling (D-brane prototypes II), Phys. Rev. D 70, 025013 (2004).

[48] Y. Isozumi, M. Nitta, K. Ohashi, and N. Sakai, Construction of Non-Abelian Walls and Their Complete Moduli Space, Phys. Rev. Lett. 93, 161601 (2004).

[49] Y. Isozumi, M. Nitta, K. Ohashi, and N. Sakai, Non-Abelian walls in supersymmetric gauge theories, Phys. Rev. D 70, 125014 (2004).

[50] M. Eto, Y. Isozumi, M. Nitta, K. Ohashi, K. Ohta, and N. Sakai, $D$-brane construction for non-Abelian walls, Phys. Rev. D 71, 125006 (2005).

[51] M. Eto, Y. Isozumi, M. Nitta, K. Ohashi, K. Ohta, N. Sakai, and Y. Tachikawa, Global structure of moduli space for BPS walls, Phys. Rev. D 71, 105009 (2005).

[52] M. Eto, M. Nitta, K. Ohashi, and D. Tong, Skyrmions from Instantons Inside Domain Walls, Phys. Rev. Lett. 95, 252003 (2005).

[53] M. Eto, T. Fujimori, Y. Isozumi, M. Nitta, K. Ohashi, K. Ohta, and N. Sakai, Non-Abelian vortices on cylinder: Duality between vortices and walls, Phys. Rev. D 73, 085008 (2006).

[54] M. Eto, T. Fujimori, M. Nitta, K. Ohashi, and N. Sakai, Domain walls with non-Abelian clouds, Phys. Rev. D 77, 125008 (2008).

[55] D. Tong, TASI lectures on solitons: Instantons, monopoles, vortices and kinks, arXiv:hep-th/0509216.

[56] M. Eto, Y. Isozumi, M. Nitta, K. Ohashi, and N. Sakai, Solitons in the Higgs phase: The moduli matrix approach, J. Phys. A 39, R315 (2006).

[57] M. Shifman and A. Yung, Supersymmetric solitons and how they help us understand non-Abelian gauge theories, Rev. Mod. Phys. 79, 1139 (2007).

[58] J. A. de Azcarraga, J. P. Gauntlett, J. M. Izquierdo, and P. K. Townsend, Topological Extensions of the Supersymmetry Algebra for Extended Objects, Phys. Rev. Lett. 63, 2443 (1989).

[59] G. W. Gibbons and P. K. Townsend, A Bogomolny Equation for Intersecting Domain Walls, Phys. Rev. Lett. 83, 1727 (1999).

[60] S. M. Carroll, S. Hellerman, and M. Trodden, Domain wall junctions are 1/4-BPS states, Phys. Rev. D 61, 065001 (2000).
[61] A. Gorsky and M. A. Shifman, More on the tensorial central charges in $N=1$ supersymmetric gauge theories (BPS wall junctions and strings), Phys. Rev. D 61, 085001 (2000).

[62] J. P. Gauntlett, R. Portugues, D. Tong, and P. K. Townsend, $D$-brane solitons in supersymmetric sigma models, Phys. Rev. D 63, 085002 (2001).

[63] M. Shifman and A. Yung, Domain walls and flux tubes in $N=2$ SQCD: $D$-brane prototypes, Phys. Rev. D 67, 125007 (2003).

[64] Y. Isozumi, M. Nitta, K. Ohashi, and N. Sakai, All exact solutions of a 1/4 Bogomol'nyi-Prasad-Sommerfield equation, Phys. Rev. D 71, 065018 (2005).

[65] N. Sakai and D. Tong, Monopoles, vortices, domain walls and $D$-branes: The rules of interaction, J. High Energy Phys. 03 (2005) 019.

[66] R. Auzzi, M. Shifman, and A. Yung, Studying boojums in $N=2$ theory with walls and vortices, Phys. Rev. D 72, 025002 (2005).

[67] M. Eto, T. Fujimori, T. Nagashima, M. Nitta, K. Ohashi, and N. Sakai, Dynamics of strings between walls, Phys. Rev. D 79, 045015 (2009).

[68] M. Eto, Y. Isozumi, M. Nitta, K. Ohashi, and N. Sakai, Instantons in the Higgs phase, Phys. Rev. D 72, 025011 (2005).

[69] M. Naganuma, M. Nitta, and N. Sakai, BPS lumps and their intersections in $N=2$ SUSY nonlinear sigma models, Gravitation Cosmol. 8, 129 (2002).

[70] M. Eto, Y. Isozumi, M. Nitta, and K. Ohashi, 1/2, 1/4, and 1/8 BPS equations in SUSY Yang-Mills-Higgs systems: Field theoretical brane configurations, Nucl. Phys. B752, 140 (2006).

[71] E. R. C. Abraham and P. K. Townsend, Intersecting extended objects in supersymmetric field theories, Nucl. Phys. B351, 313 (1991).

[72] G. Gabadadze and M. A. Shifman, $D$ walls and junctions in supersymmetric gluodynamics in the large $N$ limit suggest the existence of heavy hadrons, Phys. Rev. D 61, 075014 (2000).

[73] H. Oda, K. Ito, M. Naganuma, and N. Sakai, An exact solution of BPS domain wall junction, Phys. Lett. B 471, 140 (1999).

[74] M. A. Shifman and T. ter Veldhuis, Calculating the tension of domain wall junctions and vortices in generalized WessZumino models, Phys. Rev. D 62, 065004 (2000).

[75] K. Ito, M. Naganuma, H. Oda, and N. Sakai, Nonnormalizable zero modes on BPS junctions, Nucl. Phys. B586, 231 (2000).

[76] D. Binosi and T. ter Veldhuis, Domain wall junctions in a generalized Wess-Zumino model, Phys. Lett. B 476, 124 (2000).

[77] S. Nam and K. Olsen, Domain wall junctions in supersymmetric field theories in $D=4$, J. High Energy Phys. 08 (2000) 001.

[78] S. M. Carroll, S. Hellerman, and M. Trodden, BPS domain wall junctions in infinitely large extra dimensions, Phys. Rev. D 62, 044049 (2000).

[79] M. Naganuma, M. Nitta, and N. Sakai, BPS walls and junctions in SUSY nonlinear sigma models, Phys. Rev. D 65, 045016 (2002). 
[80] K. Kakimoto and N. Sakai, Domain wall junction in $N=2$ supersymmetric QED in four-dimensions, Phys. Rev. D 68 , 065005 (2003).

[81] M. Eto, Y. Isozumi, M. Nitta, K. Ohashi, and N. Sakai, Webs of walls, Phys. Rev. D 72, 085004 (2005).

[82] M. Eto, Y. Isozumi, M. Nitta, K. Ohashi, and N. Sakai, Non-Abelian webs of walls, Phys. Lett. B 632, 384 (2006).

[83] M. Eto, Y. Isozumi, M. Nitta, K. Ohashi, K. Ohta, and N. Sakai, $D$-brane configurations for domain walls and their webs, AIP Conf. Proc. 805, 354 (2005).

[84] M. Eto, T. Fujimori, T. Nagashima, M. Nitta, K. Ohashi, and N. Sakai, Effective action of domain wall networks, Phys. Rev. D 75, 045010 (2007).

[85] M. Eto, T. Fujimori, T. Nagashima, M. Nitta, K. Ohashi, and N. Sakai, Dynamics of domain wall networks, Phys. Rev. D 76, 125025 (2007).

[86] T. Fujimori, M. Nitta, K. Ohta, N. Sakai, and M. Yamazaki, Intersecting solitons, amoeba and tropical geometry, Phys. Rev. D 78, 105004 (2008).

[87] S. Shin, Vacua, walls and junctions in $G_{N_{F}, N_{C}}$, Nucl. Phys. B946, 114701 (2019).
[88] S. Shin, Junctions of mass-deformed nonlinear sigma models on the Grassmann manifold, J. High Energy Phys. 08 (2019) 111.

[89] T. Kim and S. Shin, Junctions of the mass-deformed nonlinear sigma models on $S O(2 N) / U(N)$ and $S p(N) /$ $U(N)$, arXiv:1909.07017.

[90] P. M. Saffin, Tiling with Almost BPS Junctions, Phys. Rev. Lett. 83, 4249 (1999).

[91] D. Bazeia and F. A. Brito, Bags, junctions and networks of BPS and non-BPS defects, Phys. Rev. D 61, 105019 (2000).

[92] A. Sen, String network, J. High Energy Phys. 03 (1998) 005.

[93] O. Aharony, A. Hanany, and B. Kol, Webs of (p,q) fivebranes, five-dimensional field theories and grid diagrams, J. High Energy Phys. 01 (1998) 002.

[94] M. Eto, M. Kawaguchi, M. Nitta, and R. Sasaki, Exhausting all exact solutions of BPS domain wall networks in arbitrary dimensions, Phys. Rev. D 101, 105020 (2020).

[95] M. Nitta, Josephson vortices and the Atiyah-Manton construction, Phys. Rev. D 86, 125004 (2012).

[96] T. Fujimori, H. Iida, and M. Nitta, Field theoretical model of multilayered Josephson junction and dynamics of Josephson vortices, Phys. Rev. B 94, 104504 (2016). 